\title{
Epigenomic and functional characterization of a core DNA methyltransferase in the human pathogen Clostridium difficile
}

Pedro H. Oliveira ${ }^{1}$, John W. Ribis ${ }^{2}$, Elizabeth M. Garrett ${ }^{3}$, Dominika Trzilova ${ }^{3}$, Alex Kim¹, Ognjen Sekulovic ${ }^{2}$, Edward A. Mead ${ }^{1}$, Theodore Pak ${ }^{1}$, Shijia Zhu ${ }^{1}$, Gintaras Deikus ${ }^{1}$, Marie Touchon $^{4,5}$, Colleen Beckford ${ }^{1}$, Nathalie E. Zeitouni ${ }^{1}$, Deena Altman ${ }^{1,6}$, Elizabeth Webster ${ }^{1}$, Irina Oussenko ${ }^{1}$, Supinda Bunyavanich ${ }^{1}$, Aneel K. Aggarwal ${ }^{7}$, Ali Bashir ${ }^{1}$, Gopi Patel ${ }^{6}$, Camille Hamula $^{6}$, Shirish Huprikar ${ }^{6}$, Eric E. Schadt ${ }^{1}$, Robert Sebra ${ }^{1}$, Harm van Bakel ${ }^{1}$, Andrew Kasarskis ${ }^{1}$, Rita Tamayo ${ }^{3}$, Aimee Shen ${ }^{2 *}$, Gang Fang ${ }^{1^{\star}}$

${ }^{1}$ Department of Genetics and Genomic Sciences, Institute for Genomics and Multiscale Biology, Mount Sinai School of Medicine, New York, New York, United States of America

${ }^{2}$ Department of Molecular Biology and Microbiology, Tufts University School of Medicine, Boston, Massachusetts, United States of America

${ }^{3}$ Department of Microbiology and Immunology, University of North Carolina at Chapel Hill School of Medicine, Chapel Hill, North Carolina, USA

${ }^{4}$ Microbial Evolutionary Genomics, Institut Pasteur, 25-28 rue du Docteur Roux, Paris, 75015, France

${ }^{5}$ CNRS, UMR3525, 25-28 rue du Docteur Roux, Paris, 75015, France

${ }^{6}$ Department of Medicine, Infectious Disease, Mount Sinai School of Medicine, New York, New York, United States of America

${ }^{7}$ Department of Pharmacological Sciences and Department of Oncological Sciences, Mount Sinai School of Medicine, New York, New York, United States of America

Email: Aimee Shen (aimee.shen@tufts.edu); Gang Fang (gang.fang@mssm.edu)

Keywords: DNA methylation; SMRT sequencing; sporulation; restriction-modification systems. 


\section{Abstract}

2 Clostridioides difficile is a leading cause of health care-associated infections. Although

3 significant progress has been made in the understanding of its genome, the epigenome of $C$.

4 difficile and its functional impact has not been systematically explored. Here, we performed

5 the first comprehensive DNA methylome analysis of $C$. difficile using 36 human isolates and

6 observed great epigenomic diversity. We discovered an orphan DNA methyltransferase with

7 a well-defined specificity whose corresponding gene is highly conserved across our dataset

8 and in all $\sim 300$ global $C$. difficile genomes examined. Inactivation of the methyltransferase

9 gene negatively impacted sporulation, a key step in C. difficile disease transmission,

10 consistently supported by multi-omics data, genetic experiments, and a mouse colonization

11 model. Further experimental and transcriptomic analysis also suggested that epigenetic

12 regulation is associated with cell length, biofilm formation, and host colonization. These

13 findings open up a new epigenetic dimension to characterize medically relevant biological

14 processes in this critical pathogen. This work also provides a set of methods for comparative

15 epigenomics and integrative analysis, which we expect to be broadly applicable to bacterial

16 epigenomics studies. 


\section{Introduction}

2 Clostridioides (formerly Clostridium) difficile is a spore-forming Gram-positive obligate

3 anaerobe and the leading cause of nosocomial antibiotic-associated disease in the

4 developed world ${ }^{1}$. Clinical symptoms of $C$. difficile infection (CDI) in humans range in

5 severity from mild self-limiting diarrhea to severe, life-threatening inflammatory conditions,

6 such as pseudomembranous colitis or toxic megacolon. Since the vegetative form of $C$.

7 difficile cannot survive in the presence of oxygen, the bacterium is transmitted via the

8 fecal/oral route as a metabolically dormant spore $^{2}$. In the intestinal environment, these

9 spores subsequently germinate into actively growing, toxin-producing vegetative cells that

10 are responsible for disease pathology ${ }^{3}$. CDI progresses in an environment of host microbiota

11 dysbiosis, which disrupts the colonization resistance typically provided by a diverse intestinal

12 microbiota $^{4}$. In the last two decades, there has been a dramatic rise in outbreaks with

13 increased mortality and morbidity due in part to the emergence of epidemic-associated

14 strains with enhanced growth ${ }^{5,6}$, toxin production ${ }^{7}$, and antibiotic resistance ${ }^{8}$. C. difficile was

15 responsible for half a million infections in the United States in 2011, with 29,000 individuals

16 dying within 30 days of the initial diagnosis ${ }^{9}$. Those most at risk are older adults, particularly

17 those who take antibiotics that perturb the normally protective intestinal microbiota.

19 Despite the significant progress achieved in the understanding of $C$. difficile physiology,

20 genetics, and genomic evolution ${ }^{10,11}$, the roles played by epigenetic factors, namely DNA

21 methylation, have not been systematically studied ${ }^{12,13}$. In the bacterial kingdom, there are

22 three major forms of DNA methylation: N6-methyladenine $(6 \mathrm{~mA}$, the most prevalent form

23 representing $\sim 80 \%)$, N4-methylcytosine $(4 \mathrm{mC})$, and 5-methylcytosine $(5 \mathrm{mC})$. Although

24 bacterial DNA methylation is most commonly associated with restriction-modification (R-M)

25 systems that defend hosts against invading foreign $D N A^{14,15}$, increasing evidence suggests

26 that DNA methylation also regulates a number of biological processes such as DNA 
1 replication and repair, cell cycle, chromosome segregation and gene expression, among

2 others $^{16-21}$. Efficient high-resolution mapping of bacterial DNA methylation events has only

3 recently become possible with the advent of Single Molecule Real-Time sequencing (SMRT-

4 seq) $)^{22,23}$, which can detect all three types of DNA methylation, albeit at different signal-to-

5 noise ratios: high for $6 \mathrm{~mA}$, medium for $4 \mathrm{mC}$, and low for $5 \mathrm{mC}$. This technique enabled the

6 characterization of the first bacterial methylomes ${ }^{24,25}$, and since then, more than 2,100 (as of

7 06/2019) have been mapped, heralding a new era of "bacterial epigenomics"26.

9 Herein, we mapped and characterized DNA methylomes of 36 human $C$. difficile isolates

10 using SMRT-seq and comparative epigenomics. We observed great epigenomic diversity

11 across $C$. difficile isolates, as well as the presence of a highly conserved methyltransferase

12 (MTase). Inactivation of this MTase resulted in a functional impact on sporulation, a key step

13 in C. difficile transmission, consistently supported by multi-omics data and genetic

14 experiments. Further experimental and integrative transcriptomic analysis suggested that

15 epigenetic regulation by DNA methylation also modulates $C$. difficile cell length, host

16 colonization and biofilm formation. Finally, the epigenomic landscape of $C$. difficile also

17 allowed us to perform a data-driven joint analysis of multiple defense systems and their

18 contribution to gene flux. These discoveries are expected to stimulate future investigations

19 along a new epigenetic dimension to characterize and potentially repress medically relevant

20 biological processes in this critical pathogen. 


\section{$1 \quad$ Results}

2 Methylome analysis reveals great epigenomic diversity in C. difficile

3 From an ongoing Pathogen Surveillance Project at Mount Sinai Medical Center, 36 C.

4 difficile isolates were collected from fecal samples of infected patients (Supplementary Table

5 1). A total of 15 different MLST sequence types (STs) belonging to clades 1 (human and

6 animal, HA1) and 2 (so-called hypervirulent or epidemic) ${ }^{27}$ are represented in our dataset

7 (Fig. 1a). Using SMRT-seq with long library size selection, de novo genome assembly was

8 achieved at high quality (Supplementary Table 1). Methylation motifs were found using the

9 SMRTportal protocol (Materials and Methods). We found a total of 17 unique high-quality

10 methylation motifs in the 36 genomes (average of 2.6 motifs per genome) (Fig. 1a,

11 Supplementary Table 2a). The large majority of target motifs were of $6 \mathrm{~mA}$ type, one motif

12 (TAAㄷTG) belonged to the $4 \mathrm{mC}$ type, and no confident $5 \mathrm{mC}$ motifs were detected

13 (Supplementary Text). Like most bacterial methylomes, $>95 \%$ of the $6 \mathrm{~mA}$ and $4 \mathrm{mC}$ motif

14 sites were methylated (Fig. 1b, Supplementary Table 2a).

16 Genomes pertaining to the same ST tend to have more similar sets of methylation motifs

17 relative to those from different STs. Those belonging to ST-2, ST-8, ST-21, and ST-110

18 showed the highest motif diversities. One $6 \mathrm{~mA}$ motif, CAAAA $\underline{A}$, was present across all

19 genomes, which led us to hypothesize that $6 \mathrm{~mA}$ methylation events at this motif, and its

20 corresponding MTase, play an important and conserved function in $C$. difficile.

\section{A DNA methyltransferase and its target motif are ubiquitous in C. difficile}

23 Motivated by the consistent presence of the methylation motif CAAAA across all the $C$.

24 difficile isolates, we proceeded to examine the encoded MTases. From the 36 genome

25 assemblies, we identified a total of 139 MTase genes (average of 3.9 per genome) (Fig. 1a,

26 Supplementary Table 2b) representing all the four major types ${ }^{28} .38$ MTases (27\%) belong 
1 to Type I R-M systems, and 100 MTases (72\%) belong to Type II (Fig. 1c). All but one of the

2 Type II MTases are solitary, i.e., devoid of a cognate restriction endonuclease (REase) (Fig.

3 1d). The least abundant MTases (1\%) belong to Type III R-M systems (Fig. 1c). A Type IV

4 (no cognate MTase) McrBC REase gene was also present in all genomes (Supplementary

5 Table 2b). $28 \%$ of MTase genes were located in mobile genetic elements (MGEs) (19\% in

6 prophages and $9 \%$ in integrative conjugative/mobile elements), while the large majority was

7 encoded in other chromosomal regions (Fig. 1e, Supplementary Tables 2b-d). No MTase

8 genes were found in plasmids. We further found multiple additional defense systems (e.g.,

9 abortive infection systems, CRISPR-Cas, toxin-antitoxin), and performed an integrative

10 analysis with R-M systems in relation to host defense and gene flux (Supplementary Figs.

11 1a-e, Supplementary Fig. 2, Supplementary Tables 3a-f, Supplementary Text), such as that

12 involving phages (Supplementary Fig. 3, Supplementary Table 3g, Supplementary Text).

14 Consistent with the presence of a highly conserved CAAAA $\underline{A}$ motif, we identified a Type II

$156 \mathrm{~mA}$ solitary DNA MTase (577 aa) present across isolates (Fig. 1f, Supplementary Table

16 2b). This MTase is encoded by $C D 2758$ in $C$. difficile 630, a reference strain that was

17 isolated from a $C$. difficile outbreak in Switzerland ${ }^{10,29}$. Here we have named CD2758 as

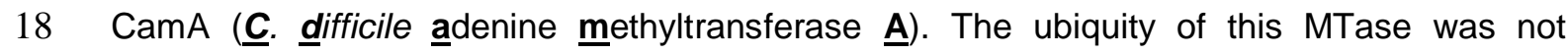

19 restricted to the 36 isolates, as we were able to retrieve orthologs in a list of $\sim 300$ global $C$.

20 difficile isolates from GenBank (Supplementary Table 4). REBASE also showed functional

21 orthologs of camA only in very few other Clostridiales and Fusobacteriales (Supplementary

22 Figs. 4a, b), suggesting that this MTase is fairly unique to $C$. difficile. The genomic context of

23 camA is largely conserved across strains (Supplementary Fig. 4c), located 25 kb upstream

24 of the S-layer biogenesis locus (Supplementary Fig. 4d). Several of the genes flanking camA

25 (including itself) are part of the $C$. difficile core-genome (see below), suggesting that they

26 may play biological roles fundamental to $C$. difficile. Hence, CamA is a solitary Type II $6 \mathrm{~mA}$

27 MTase that specifically recognizes the CAAAAA motif and is ubiquitous and fairly unique to

28 C. difficile. 


\section{Inactivation of camA reduces sporulation levels in vitro}

3 To discover possible functional roles of CamA, we searched among published transposon

4 sequencing studies of $C$. difficile. From a recent study analyzing $C$. difficile gene essentiality

5 and sporulation, we found a homolog of camA (R20291_2646) among 800 genes whose

6 mutation impacted sporulation in the 027 isolate R2029130. Given the critical role of

7 sporulation in the persistence and dissemination of $C$. difficile in humans and hospital

8 settings $^{31}$, we decided to test if camA inactivation could reduce spore purification efficiencies

9 in the 630 6 erm strain background as seen for R20291_2646 transposon mutants. We used

10 allele-coupled exchange ${ }^{32}$ to construct an in-frame deletion in $630 \Delta$ erm camA and to

11 complement $\triangle$ camA with either wild-type $\operatorname{cam} A$ or a variant encoding a catalytic site

12 mutation (N165A) of the MTase in single copy from the pyrE locus (Supplementary Fig. 5a;

13 Supplementary Tables 5a, b; Supplementary Materials and Methods; Supplementary Text).

14 We observed that spore purification efficiencies decreased by $\sim 50 \%$ in the mutant relative to

15 wild type (Fig. 2a, $P<10^{-2}$, ANOVA, Tukey's test). Complementation of $\triangle$ camA with the wild

16 type, but not the catalytic mutant construct, restored spore purification efficiencies to values

17 similar to those observed in wild-type cells (Fig. 2a, Supplementary Table 5c). No

18 differences in growth were observed between wild-type and mutant strains (Supplementary

19 Fig. 5b). Hence, this complementation experiment supports that the loss of methylation

20 events by CamA, rather than the loss of non-catalytic roles of this protein, leads to the

21 decrease in spore yield.

23 The diminished spore purification efficiencies observed in the $\triangle$ camA mutants could be due

24 to reduced number of cells that induce sporulation or defects in spore assembly ${ }^{33}$. Visual

25 inspection of samples before and after spore purification on a density gradient revealed

26 qualitatively lower levels of mature, phase-bright spores (Supplementary Fig. 5c). Since

27 purified wild-type and $\triangle$ camA spores had similar levels of chloroform resistance

28 (Supplementary Fig. 5d), and wild-type and $\Delta$ camA spores germinated with similar efficiency 
1 when plated on media containing germinant (Supplementary Fig. 5e), the reduced spore

2 purification efficiencies of the MTase mutants likely reflect a defect in sporulation initiation

3 rather than the sporulation process itself.

5 Consistent with this hypothesis, fewer $\triangle$ camA cells were qualitatively observed to be

6 sporulating in phase-contrast microscopy analyses relative to wild type (Fig. 2b). To

7 determine whether loss of CamA impairs the frequency rather than the progression of

8 sporulation, we visualized and quantified the number of sporulating cells at different stages

9 of spore assembly using the FM4-64 membrane stain and Hoechst nucleoid $\operatorname{stain}^{34}$ (Fig. 2c).

11 While similar numbers of wild-type and $\Delta$ camA cells were observed at asymmetric division

12 (the first morphological stage of sporulation), at the $9 \mathrm{~h}$ time point, $50 \%$ fewer $\triangle$ camA cells

13 had initiated engulfment. Furthermore, $11 \mathrm{~h}$ after sporulation induction, $\sim 2$-fold more $\Delta$ camA

14 cells were at the asymmetric division stage relative to wild type, whereas $50 \%$ fewer $\Delta$ camA

15 cells had completed engulfment. Since similar numbers of sporulating cells were observed

16 between wild type and $\triangle$ camA at the $11 \mathrm{~h}$ timepoint, $\triangle$ camA's sporulation defect appears to

17 arise from fewer cells progressing beyond asymmetric division rather than a defect in

18 sporulation induction.

19 To confirm that loss of CamA leads to a decrease in the number of cells producing functional

20 spores, we compared the ability of $\triangle$ camA to form heat-resistant spores capable of

21 germinating and outgrowing using a heat resistance assay ${ }^{35}$. The $\Delta c a m A$ mutant and the

22 catalytic mutant complementation strain produced $\sim 50 \%$ fewer heat-resistant spores than

23 wild-type and the wild-type complementation strain (Supplementary Fig. 5e, $P<10^{-3}$,

24 ANOVA, Tukey's test). Taken together, these findings suggest that CAAAA $\underline{A}$ methylation

25 enhances sporulation in vitro. This functional difference prompted us to perform a

26 comprehensive methylome and transcriptome analysis of the wild-type and MTase mutant

27 strains. 
1 Comparative analysis of CAAAAA sites across $C$. difficile genomes

2 The $C$. difficile genome has an average of $7,721( \pm 197$, sd) CAAAAA motif sites

3 (Supplementary Table 6a). Adjusted by the k-mer frequency of the AT-rich $C$. difficile

4 genome (70.9\%) using Markov models ${ }^{36}$ (Materials and Methods), CAAAAA motif sites are

5 significantly under-represented in the chromosome, particularly in intragenic regions

6 (Supplementary Fig. 6a, Supplementary Table 6b). To evaluate if specific chromosomal

7 regions are enriched or depleted for this motif, we used a multi-scale signal representation

8 (MSR) approach ${ }^{37}$ (Materials and Methods). Briefly, MSR uses wavelet transformation to

9 examine the chromosome at a succession of increasing length scales by testing for

10 enrichment or depletion of a given genomic signal. While scale values $<10$ are typically

11 associated with regions $<100 \mathrm{bp}$, genomic regions enriched for CAAAA $\underline{A}$ sites at scale

12 values $>20$ corresponding to segments larger than $1 \mathrm{~kb}$ (i.e. gene and operon scale) were

13 observed and included genes related to sporulation and colonization (Fig. 3a, regions A-E;

14 Supplementary Tables 6c, d): stage 0 (spo0A), stage III (spollIAA-AH), and stage IV

15 (spolVB, sigK) of sporulation, membrane transport (PTS and ABC-type transport systems),

16 transcriptional regulation (e.g., iscR, fur), and multiple cell wall proteins (CWPs).

18 To further characterize CAAAAA motif sites, we built upon our large collection of $C$. difficile

19 methylomes by categorizing these motif sites on the basis of their positional conservation

20 across genomes. We performed whole genome alignment of 37 C. difficile genomes (36

21 isolates + C. difficile 630 as reference) (Materials and Methods, Supplementary Dataset 1),

22 and classified each motif position in the alignment as either: (1) conserved orthologous

23 (devoid of SNPs or indels); (2) variable orthologous (in which at least one genome contains

24 a SNP or indel); and (3) non-orthologous (Fig. 3b). We found a total of 5,828 conserved

25 orthologous motif positions, 1,050 variable orthologous positions (885 with SNPs and 165

26 with indels), and an average of 843 non-orthologous positions per genome (Supplementary

27 Table 6e). The latter were, as expected, largely mapped to MGEs. Among orthologous 
1 positions, the variable ones represent a particularly interesting subset to study, since they

2 contribute to variations of CAAAA $\underline{A}$ sites across genomes with subsequent methylation

3 abrogation (Supplementary Table 6f). We used DAVID gene enrichment analysis and found

4 cytoplasm- (e.g.: pheA, fdhD, ogt1, spolVA) and motility-related genes (e.g.: fliZ, fliN, fliM,

$5 \mathrm{flgL}$ ) to over-represent orthologous variable CAAAAA positions (FDR $<5 \%$, Fig. $3 \mathrm{c}$ ).

6 Concomitantly, the regions with the highest density of orthologous variable positions were

7 found at the S-layer locus region (region D, Fig. 3a) and between positions $0.31-0.33 \mathrm{Mb}$,

8 which is rich in flagellar genes. The very large number and dispersion of conserved

9 orthologous positions precluded a similar functional analysis. To test whether homologous

10 recombination (HR) contributes to the cross-genome variation of CAAAA $\underline{A}$ motif sites

11 located in the core-genome, we also performed a systematic analysis of such events

12 (Supplementary Figs. 6b-d, Supplementary Table 6g, Supplementary Text) and found that

$13 \mathrm{HR}$ tracts indeed over-represent $\left(\mathrm{O} / \mathrm{E}=1.40, P<10^{-3}\right.$; Chi-square test) orthologous variable

14 CAAAAA motif positions, while the core-genome without HR tracts underrepresents them

$15\left(\mathrm{O} / \mathrm{E}=0.89, P<10^{-3}\right.$; Chi-square test) (Supplementary Figs. $\left.6 \mathrm{e}, \mathrm{f}\right)$.

17 Collectively, genome-wide distribution analyses and across-genome comparative analyses

18 suggest that CAAAA $\underline{\text { sites }}$ are enriched in regions harboring genes related to sporulation

19 and colonization, orthologous variable CAAAA $\underline{\text { A }}$ positions are enriched in regions harboring

20 genes related to cytoplasm- and motility-related genes, and the former's variability is at least

21 partially fueled by HR.

\section{Non-methylated CAAAAA motif sites are enriched in regulatory elements}

24 DNA methylation is highly motif driven in bacteria; i.e. in most cases, $>95 \%$ of the 25 occurrence of a methylation motif is methylated. However, a small fraction of methylation 26 motif sites can be non-methylated. The on/off switch of DNA methylation in a bacterial cell 27 can contribute to epigenetic regulation as a result of competitive binding between DNA 28 MTases and other DNA binding proteins (e.g. transcription factors, TFs) as previously 
1 described for $E$. coli $i^{21,38-40}$. Previous bacterial methylome studies analyzing one or few

2 genomes usually have insufficient statistical power to perform a systematic interrogation of

3 non-methylated motifs sites. Building on our rich collection of $36 \mathrm{C}$. difficile methylomes, we

4 performed a systematic detection and analysis of non-methylated CAAAA $\underline{A}$ sites. To classify

5 a CAAAA $\underline{A}$ site as non-methylated, we adopted stringent filtering criteria at interpulse

6 duration ratio (ipdR) and sequencing coverage, and found an average of 21.5 non-

7 methylated CAAAA $\underline{A}$ motif sites per genome (Supplementary Fig. 7a; Supplementary Table

8 7a). Non-methylated motif sites were found dispersed throughout the full length of the $C$.

9 difficile genome, yet were overrepresented in orthologous variable and non-orthologous

10 CAAAA $\underline{A}$ positions $(\mathrm{O} / \mathrm{E}=$ respectively 1.51 and 1.49$)$ and underrepresented in orthologous

11 conserved CAAAAA positions (O/E 0.84) $\left(P<10^{-4}\right.$; Chi-square test). This is consistent with

12 the idea that variable positions are more likely to be non-methylated to provide breadth of

13 expression variation. Most of the non-methylated positions ( $85.4 \%$ of 245 ) failed to conserve

14 such status in more than three genomes at orthologous positions (Fig. 4a, Supplementary

15 Table $7 a)$, while a minor percentage of positions (5.5\%) remained non-methylated in at least

16 one third of the isolates, suggesting that competitive protein binding is expected to be more

17 active in certain genomic regions (Fig. 4a), e.g. upstream of the pathogenicity locus (PaLoc)

18 (position 786,216$)$, in prophage genes $(1,593,616)$, within the atp operon $(3,430,190)$, and in

19 the xylose operon $(3,561,672)$ (Supplementary Fig. $7 b)$.

21 The non-methylated CAAAAA positions detected across the 36 C. difficile genomes allowed 22 a systematic search for evidence of overlap with TF binding sites (TFBSs) and transcription 23 start sites (TSSs). To test this, we queried our genomes for putative binding sites of 21 TFs 24 pertaining to 14 distinct families (Supplementary Table $7 \mathrm{~b}$; Materials and Methods) and 25 found overlaps between prominent peaks of non-methylated CAAAA $\underline{A}$ positions (Fig. 4a) 26 and the TFBSs of CodY and XyIR (Fig. 4b, Supplementary Fig. 7b, Supplementary Table

27 7c). Performing the analysis at the genome level, both CodY and XylR binding sites showed 28 significant enrichment $\left(P<10^{-3}\right.$, Mann-Whitney-Wilcoxon test) for non-methylated CAAAA $\underline{A}$ 
1 (Fig. 4c; Supplementary Fig. 7c). In a similar enrichment analysis using 2,015 TSSs

2 reconstructed from RNA-seq data coverage ${ }^{41}$ (Materials and Methods; Supplementary Table

3 7d), we found a genome-level enrichment: non-methylated CAAAA $\underline{A}$ sites preferentially

4 overlapped with TSSs (Figs. 4d, e; Supplementary Figs. 7d, e; $P<10^{-3}$, Mann-Whitney-

5 Wilcoxon test,).

6

7 In addition to the on/off epigenetic switch driven by competitive binding between the MTase

8 and other DNA binding proteins at CAAAA $\underline{A}$ sites, we hypothesized that epigenetic

9 heterogeneity within a clonal population could also stem from DNA replication errors at

10 CAAAA $\underline{A}$ sites, especially because homopolymer tracts are expected to be more error-

11 prone $^{42}$. To test this hypothesis, we investigated if CAAAAA had a larger than expected

12 frequency of mutated reads (compared to baseline sequencing errors). Specifically, we

13 tested if CAAAA $\underline{A}$ sites in $C$. difficile are more error prone during DNA replication, compared

14 to the control motifs TAAAAA or GAAAAA (here called KAAAAA). To account for the

15 confounding effect of mutation rate at different sequence contexts, we further computed the

$16 \%$ mutated reads (SNPs + indels) in four distinct species with a broad dispersion of mutation

17 rates (Mycobacterium tuberculosis $<E$. coli $\approx$ C. difficile $<$ Helicobacter pylori). Interestingly,

18 we indeed found a significantly higher $\%$ of mutated reads mapping to CAAAA $\underline{A}$ sites

19 compared to KAAAAA sites in C. difficile (Fig. 4f, Supplementary Fig. 7f), while this

20 difference was not observed in any of the control genomes. The observation of increased

21 mutagenesis at $6 \mathrm{~mA}$ target sites is in line with recent data obtained in Neisseria

22 meningitidis ${ }^{43}$ and is worth further investigation in future work.

24 Collectively, these results highlight two types of variations that affect CAAAA $\underline{A}$ methylation

25 status in $C$. difficile: (i) on/off epigenetic switch of CAAAA $\underline{A}$ sites preferentially overlapping

26 with putative TFBSs and TSSs, and (ii) higher mutation rates at the CAAAAA motif sites that

27 contribute to cell-to-cell heterogeneity. 
1 Loss of CAAAAA methylation impacts transcription of multiple gene

\section{2 categories including sporulation}

3 To study the functional significance of methylation at CAAAAE sites, we used RNA-seq to

4 compare the transcriptomes of wild-type $C$. difficile $630 \Delta e r m$ with that of $\triangle$ camA both in

5 liquid medium (exponential and stationary growth stage) and following sporulation induction

6 (9 and 10.5 h) (Supplementary Fig. 8, Supplementary Table 8a, Materials and Methods). Of

7 the 3,896 genes annotated in C. difficile $630,36-361(0.9-9.3 \%$, depending on the time

8 point) were differentially expressed (DE) at a 5\% FDR and $\left|\log _{2} F C\right|>1$ (2-fold change in

9 gene expression) (Fig. 5a, Supplementary Tables 8b-d). DE genes in $\triangle$ camA relative to wild

10 type appeared to have a significant enrichment in CAAAAㄹ motif sites compared to non-DE

11 genes $\left(P<10^{-2}\right.$, Mann-Whitney-Wilcoxon test) in broth culture; a qualitatively similar trend

12 was also observed during sporulation (Supplementary Fig. 9a). Consistent with our finding

13 that loss of CamA reduces spore formation, the transcriptome analyses revealed that 118

14 and 120 genes previously identified as being induced during sporulation ${ }^{44,45}$ were expressed

15 at $\geq 50 \%$ lower levels in $\Delta$ camA cells relative to wild type at the 9 and $10.5 \mathrm{~h}$ timepoints,

16 respectively (Supplementary Table $8 b)$.

18 The transcriptional program that mediates sporulation in $C$. difficile is controlled by a master

19 transcriptional activator, Spo0A, and four sporulation-specific sigma factors, $\sigma^{\mathrm{F}}, \sigma^{\mathrm{E}}, \sigma^{\mathrm{G}}$ and

$20 \sigma^{\mathrm{K}}$. These factors activate distinct regulons that ultimately lead to the assembly of functional

21 spores $^{37,38}$ (Fig. 5b). Activated Spo0A induces the expression of genes encoding $\sigma^{\mathrm{F}}$, $\sigma^{\mathrm{E}}$, and

$22 \sigma^{G}$ as well as factors required for asymmetric division and the post-translational activation of

23 the early-stage sporulation sigma factors, $\sigma^{F}$ and $\sigma^{E}$ (Fig. 5b). $\sigma^{F}$ is the first sporulation-

24 specific sigma factor to be fully activated, and it only becomes active in the forespore after

25 asymmetric division is completed ${ }^{46}$. Activated $\sigma^{F}$ subsequently induces the transcription of

26 genes whose products mediate $\sigma^{G}$ activation in the forespore and partially mediates $\sigma^{\mathrm{E}}$

27 activation in the mother cell ${ }^{47}$. Activated $\sigma^{\mathrm{E}}$ induces the transcription of $\operatorname{sig}^{48}$ and factors 
1 required for the excision of a prophage-like element from the sigK gene ${ }^{49}$. Thus, $C$. difficile

2 sporulation is controlled by a transcriptional hierarchy that is coupled to morphological

3 events such that downstream sigma factors $\left(\sigma^{G}\right.$ and $\left.\sigma^{K}\right)$ depend on the activation of

4 upstream sigma factors $\left(\sigma^{F}\right.$ and $\left.\sigma^{\mathrm{E}}\right)$. Notably, genes in the regulons of all four sporulation-

5 specific sigma factors were under-expressed in $\triangle$ camA relative to wild type, whereas a

6 relatively small subset of SpoOA regulon genes exhibited this pattern of regulation

7 (Supplementary Fig. 9b, Supplementary Table 8e). These observations suggested that loss

8 of CamA reduces spore formation by targeting events early on during sporulation.

10 To gain further insight into the regulatory stage of sporulation that CamA-mediated DNA

11 methylation specifically impacts, we used qRT-PCR to analyze the expression of genes

12 encoding SpoOA, the sporulation-specific sigma factors ${ }^{44,50}$, and their individual regulons ${ }^{51-53}$.

13 These analyses were conducted on a separate set of RNA samples harvested at 9 and $11 \mathrm{~h}$

14 following sporulation induction. Consistent with our RNA-Seq analyses, Spo0A regulon

15 genes, spoOA, sig $F$, and $\operatorname{sig} E^{44,51}$, were expressed at similar levels between wild type and

$16 \Delta$ camA at both 9 and $11 \mathrm{~h}$, implying that the $\triangle$ camA mutant activates Spo0A at levels similar

17 to wild type. In contrast, $\sigma^{F}$ and $\sigma^{\mathrm{E}}$ regulon genes, spolIQ and spolVA $A^{44,54}$, respectively, were

18 under-expressed in $\triangle$ camA relative to wild type (Fig. 5b). Reduced SpollQ and SpoIVA

19 levels were observed in $\triangle c a m A$ by western blot, confirming the transcriptional analyses

20 (Supplementary Fig. 9c). Based on the hierarchical organization of the sporulation regulatory

21 cascade, these results implicate $\sigma^{F}$ activation as the earliest sporulation stage affected by

22 CamA. This conclusion is supported by our morphological analyses, since fewer $\Delta$ camA

23 cells initiate and complete engulfment relative to wild type, and engulfment requires both $\sigma^{F}$ -

24 and $\sigma^{E}$-dependent gene products ${ }^{55}$. Notably, similar numbers of $\Delta$ camA and wild-type cells

25 are sporulating based on these morphological analyses (Fig. 2c), consistent with the similar

26 levels of spoOA observed in WT and $\triangle$ camA (Fig. 5b). While a small subset of Spo0A

27 regulon genes are under-expressed in $\triangle$ camA cells, at least some of these genes are dually 
1 regulated by SpoOA and $\sigma^{F}$. For example, transcription of $s p o l / R^{52}$, which encodes a

2 signaling protein required for $\sigma^{\mathrm{E}}$ activation, is activated by both $\mathrm{SpoOA}$ and $\sigma^{\mathrm{F} 44,47}$.

3

4 In vivo impacts of the camA mutation

5 To test whether the sporulation defect of $\Delta$ camA impacts $C$. difficile infection or transmission,

6 we analyzed the effect of the $\Delta$ camA mutation in an established mouse model of infection in

7 which a cocktail of antibiotics is used to sensitize the animals to $C$. difficile infection ${ }^{56}$. When

8 inoculated with $630 \Delta$ erm strains, antibiotic-treated mice typically do not develop fulminant

9 disease and instead serve as a model of intestinal colonization and persistence by $C$.

10 difficile ${ }^{57,58}$. Groups of mice (6 males, 6 females) were inoculated by oral gavage with

$1110^{5}$ spores of the three genotypes: wild type, $\triangle$ camA, and $\triangle$ camA-C. No mortality was

12 observed at the given doses of $C$. difficile spores as expected. Fecal samples were collected

13 every $24 \mathrm{~h}$ for seven days. All three $C$. difficile strains reached comparable levels in feces at

14 days 1 and 2 post-inoculation, indicating that they germinate and establish colonization

15 equally (Fig. 6a, Supplementary Materials and Methods). As expected, CFU levels

16 decreased steadily from day 2 post-inoculation to day 7. However, the $\triangle$ camA mutant

17 showed CFU levels 10-100 times lower than those observed in the wild-type and

18 complemented strains throughout this time frame (ANOVA, $P<10^{-4}$ ). The bacteria declined

19 below the limit of detection in the feces 6 days post-inoculation for the MTase mutant, while

20 they remained detectable at days 6 and 7 for the wild-type and complemented strains.

22 To test whether loss of CamA leads to defects in virulence, we compared $\triangle$ camA and wild

23 type in a hamster model of infection. Clindamycin-treated golden Syrian hamsters are highly

24 susceptible to the effects of the $C$. difficile toxins and thus represent a model of acute

25 disease. Groups of 6 hamsters ( 3 male, 3 female) were inoculated by oral gavage with

$2610^{3}$ spores of the wild-type, $\triangle c a m A$, and $\triangle$ camA-C strains. These $C$. difficile strains elicited

27 diarrheal symptoms and weight loss in this model, and we observed no difference in animal 
1 survival times post inoculation (Fig. 6b, Supplementary Materials and Methods). This result

2 is consistent with the observation that the wild-type, $\triangle \operatorname{cam} A$, and $\triangle \operatorname{cam} A-C$ strains exhibit no

3 differences in toxin gene expression (Supplementary Table 8a) and produce comparable

4 levels of TcdA in vitro (Supplementary Fig. 9d). Together, these data indicate that CAAAA $\underline{A}$

5 methylation by CamA does not influence toxin-mediated aspects of $C$. difficile pathogenesis

6 but instead impacts $C$. difficile's ability to persist within the host intestinal tract.

\section{Additional functional impacts of the camA mutation}

9 Considering the high conservation of camA across $C$. difficile genomes, we asked if some

10 additional phenotypes could be impacted by the gene's inactivation. While analyzing images

11 of sporulating $C$. difficile, we noticed that $\triangle$ camA mutant cells appeared to be shorter on

12 average than wild-type cells. To test this possibility, we measured the lengths of wild-type

13 and $\triangle$ camA cells during log-phase and stationary-phase growth in broth culture and during

14 sporulation ( 9 and $11 \mathrm{~h}$ time points). These analyses revealed that $\Delta$ camA cells were $\sim 15 \%$

15 shorter than wild-type cells $(P<0.001$, one-way ANOVA, Fig. 6c, d) even though no

16 difference in cell growth was observed (Supplementary Fig. 5b). Interestingly, genes

17 encoding putative cell wall remodeling enzymes, spoVD, cwp16, and cwp17, were over-

18 expressed in the $\triangle$ camA mutant relative to wild type during growth in broth culture

19 (Supplementary Fig. 9e).

21 In addition to the above experimental characterization, we attempted to build on the RNA-

22 seq data further beyond the sporulation phenotype, with a special focus on biological

23 processes critical to $C$. difficile infection. Specifically, we performed an overlap analysis

24 between the list of DE genes from our RNA-seq data (wild type vs. $\triangle$ camA mutant; four

25 different time points) and those from published studies focusing on the colonization and

26 infection by this pathogen (Materials and Methods). Out of the total 20 pairwise overlap

27 analyses, we observed 9 significant overlaps $(P<0.01$ with Bonferroni correction) between

28 our dataset and those of others (Supplementary Table 8f). First, DE genes in the $\Delta$ camA 
1 mutant (sporulation phases) had a significant overlap to DE genes in conditions favoring the

2 production of biofilm on a solid substrate $^{59}$ (Fig. 6e, Supplementary Table 8f) $(\mathrm{O} / \mathrm{E}=1.3-1.6$,

$3 P<10^{-9}$, Chi-Square test). Motivated by this significant overlap, we performed biofilm assays

4 using crystal violet staining of adherent biofilm biomass, and made consistent observation

5 that the $\triangle$ camA mutant produced more biofilm than wild type (Fig. 6f). These results suggest

6 that methylation inhibits the expression of genes that promote biofilm formation, e.g.,

7 encoding factors involved in the adherence to a surface and/or stabilizing interbacterial

8 interactions. The differences in biofilm production between $\triangle$ camA and $\triangle \operatorname{camA} / N 165 A$ could

9 be explained if the latter retained some DNA binding ability capable of altering transcription

10 of some genes even in the absence of methylation. Second, by comparing DE genes in

$11 \Delta$ camA relative to wild type with genes $\mathrm{DE}$ during infection in different murine gut

12 microbiome compositions ${ }^{60}$, we found a statistically significant overlap, particularly genes

13 expressed at stationary stage $\left(\mathrm{O} / \mathrm{E}=1.4, P<10^{-6}\right.$, Chi-Square test) (Supplementary Fig. 10a,

14 Supplementary Table 8f). Lastly, using DE genes obtained from murine gut isolates at

15 increasing time points after infection ${ }^{61}$, we found significant overlaps with DE genes in the

$16 \Delta$ camA mutant $\left(\mathrm{O} / \mathrm{E}=1.5, \quad P<10^{-4}\right.$, Chi-Square test) (Supplementary Fig. 10b,

17 Supplementary Table 8f). Collectively, these integrative overlap analyses provide additional

18 evidence that DNA methylation events by CamA may directly and/or indirectly affect the

19 expression of multiple genes involved in the in vivo colonization and biofilm formation of $C$.

20 difficile and inspire future work to elucidate the mechanisms underlying the functional roles

21 of CAAAAA methylation in C. difficile pathogenicity.

\section{Discussion}

24 C. difficile is responsible for one of the most common hospital-acquired infections and

25 classified by the US Centers for Disease Control and Prevention as an urgent healthcare

26 risk with significant morbidity and mortality ${ }^{9}$. Because CDI is spread by bacterial spores

27 found within feces, extensive research has been devoted to better understand the genome 
1 of this critical pathogen and its sporulation machinery. To address these common goals, we

2 performed the first comprehensive characterization of the DNA methylation landscape

3 across a diverse collection of clinical isolates. During our epigenome analysis, we identified

4 an 6mA MTase $(\mathrm{camA})$ conserved across all isolates (and in another $\sim 300$ published $C$.

5 difficile genomes) sharing a common methylation motif (CAAAA $\underline{A})$. Inactivation of the gene

6 encoding this MTase resulted in a sporulation defect in vitro (Fig. 2). This represents, to the

7 best of our knowledge, the first time that DNA methylation has been found to impact

8 sporulation in any bacterium, opening a new dimension of study in C. difficile. Infection

9 studies using the mouse model indicate a role for CamA in the persistence of $C$. difficile in

10 the intestinal tract. Since enumeration of $C$. difficile recovered in feces of the infected

11 animals reflects the number of $C$. difficile spores in the gut, the reduced burden of $\triangle$ cam $A$ in

12 the mouse may be due to the mutant's defect in sporulation, as the ability to form spores

13 was previously shown to be important for persistence ${ }^{2}$. That $\Delta$ camA exhibited virulence

14 comparable to the wild type in the hamster model suggests that DNA methylation does not

15 impact toxin-mediated disease. However, due to the pleiotropic nature of the MTase it

16 remains possible that multiple factors contribute to the more pronounced effect observed in

17 the mouse model.

19 The highly conserved camA and its flanking genes across $C$. difficile genomes suggest that

20 additional phenotypes may be regulated by CamA beyond sporulation. Consistently,

21 CAAAA $\underline{A}$ sites were overrepresented in a set of regions enriched in genes with functions

22 linked to sporulation, motility, and membrane transport. Further supporting a broader

23 regulatory network of CamA, is that its loss reduces cell length and results in statistically

24 significant overlap between transcriptional signatures identified in our study (wild type vs

$25 \Delta$ camA mutant) and those of others observed during the in vivo colonization and biofilm

26 formation (Fig. 5b, Supplementary Figs. 7b, c). 
1 The fact that camA is a solitary MTase gene without a cognate restriction gene further

2 supports a view that widespread methylation in bacteria has a functional importance beyond

3 that attributed to R-M systems. Previously, the most extensively characterized $6 \mathrm{~mA}$ MTase

4 was Dam targeting GATC in E. coli. Dam plays multiple important functions and is essential

5 in some pathogens ${ }^{21}$. However, since it is conserved in the large diversity of $\mathrm{Y}^{-}$

6 proteobacteria, it was not considered a promising drug target. In contrast, the uniqueness of

7 camA in all C. difficile genomes and in just a few Clostridiales makes it a promising drug

8 target that may inhibit $C$. difficile in a much more specific manner, which is particularly

9 relevant since gut dysbiosis potentiates $C$. difficile infection ${ }^{62,63}$. In addition, since this MTase

10 seemingly does not impact the general fitness of $C$. difficile ${ }^{30}$, a drug specifically targeting it

11 may be developed with a lower chance for resistance.

13 Considering the large number of genes differentially expressed in the $\triangle$ camA mutant, the

14 functional impact of CAAAAA methylation is likely mediated by multiple genes that are either

15 directly regulated by DNA methylation or indirectly regulated by a transcriptional cascade.

16 Mechanistically, DNA methylation can either activate or repress a gene depending on other

17 DNA binding proteins that compete with DNA MTases ${ }^{16,17,21,64}$, so the competition between

18 transcription factors and MTases may form an epigenetic switch to turn on/off a gene.

20 With more than 2,200 bacterial methylomes published to date, it is becoming increasingly

21 evident that epigenetic regulation of gene expression is highly prevalent across bacterial

22 species. Despite the exciting prospects for studying epigenetic regulation, our ability to

23 comprehensively analyze bacterial epigenomes is limited by a bottleneck in integratively

24 characterizing methylation events, methylation motifs, transcriptomic data, and functional

25 genomics data. In this regard, this work represents the first comprehensive comparative

26 analysis of a large collection of a single bacterial species and thus provides a detailed

27 roadmap that can be used by the scientific community to leverage the current status quo of

28 epigenetic analyses. 


\section{$1 \quad$ Materials and Methods}

\section{Data and code availability}

3 Genome assemblies are available via NCBI under BioProject ID PRJNA448390. RNA-Seq

4 data has been submitted to the NCBI Sequence Read Archive (SRA) under project

5 PRJNA445308. Scripts and a tutorial supporting all key analyses of this work will be made

6 publicly available at http://github.com/fanglab/ upon the acceptance of the manuscript.

$8 \quad$ Clostridium difficile isolates and culture

936 clonal C. difficile isolates from CDI fecal samples were obtained using protocols

10 developed in an ongoing Pathogen Surveillance Program at Mount Sinai Hospital

11 (Supplementary Table 1). Additionally, 9 fully sequenced and assembled $C$. difficile

12 genomes were retrieved from Genbank Refseq (ftp://ftp.ncbi.nih.gov/genomes, last

13 accessed in November 2016) (Supplementary Table 1). Raw sequencing data from global

14 and UK collections comprising 291 C. difficile 027/BI/NAPI genomes were used ${ }^{11}$

15 (Supplementary Table 4). C. difficile positive stool samples were frozen at $-80{ }^{\circ} \mathrm{C}$ prior to

16 analysis. All stool samples underwent culture for $C$. difficile using ethanol shock culture

17 method, adapted from Griffiths et al. Briefly, approximately $80 \mathrm{mg}$ of solid stool (50 $\mu$ liquid

18 stool samples) was added to $0.5 \mathrm{ml}$ of $70 \%$ ethanol wash and the sample was vortex mixed

19 and incubated at room temperature for $20 \mathrm{~min}$. A loopful was then cultured onto $C$. difficile

20 selective agar (CDSA, Becton Dickinson, Franklin Lakes, NJ) and the plates were incubated

21 anaerobically at $37^{\circ} \mathrm{C}$ for up to $72 \mathrm{~h}$. A single colony was subcultured onto a Trypticase ${ }^{\mathrm{TM}}$

22 soy agar with 5\% defibrinated sheep blood plate (TSA II ${ }^{\mathrm{TM}}$, Becton Dickinson, Franklin

23 Lakes, $\mathrm{NJ}$ ) and incubated anaerobically at $37^{\circ} \mathrm{C}$ for $48 \mathrm{~h}$, after which colonies giving the

24 characteristic $C$. difficile odor and fluorescence under UV illumination were obtained and

25 confirmed by MALDI on a Brucker biotyper. For long-term storage, individual colonies were

26 emulsified in tryptic soy broth containing $15 \%$ glycerol and stored at $-80^{\circ} \mathrm{C}$. 


\section{$1 \quad$ Single-molecule real-time (SMRT) sequencing}

2 Primer was annealed to size-selected (>8 kb) SMRTbells with the full-length libraries $(80 \stackrel{\circ}{\circ}$

3 for $2 \mathrm{~min}$ and $30 \mathrm{~s}$ followed by decreasing the temperature by $0.1{ }^{\circ} \mathrm{C}$ increments to $25 \stackrel{\circ}{\circ} \mathrm{C}$ ).

4 The polymerase-template complex was then bound to the P6 enzyme using a ratio of 10:1

5 polymerase to SMRTbell at $0.5 \mathrm{nM}$ for $4 \mathrm{~h}$ at $30{ }^{\circ} \mathrm{C}$ and then held at $4{ }^{\circ} \mathrm{C}$ until ready for

6 magbead loading, prior to sequencing. The magnetic bead-loading step was conducted at 4

$7 \quad{ }^{\circ} \mathrm{C}$ for 60 min per manufacturer's guidelines. The magbead-loaded, polymerase-bound,

8 SMRTbell libraries were placed onto the RSII machine at a sequencing concentration of

$9 \quad 125-175 \mathrm{pM}$ and configured for a 240 min continuous sequencing run.

\section{De novo genome assembly and motif discovery}

12 The RS_HGAP3 protocol was used for de novo genome assembly, followed by custom

13 scripts for genome finishing and annotation. RS_Modification_and_Motif_Analysis.1 was

14 used for de novo methylation motif discovery. A custom script was used to examine each

15 motif to ensure its reliable methylation states. In brief, variations of a putative motif are

16 examined by comparing the ipdR distribution of each variation with non-methylated motifs.

18 Identification of R-M systems

19 Identification of R-M systems was performed as previously described ${ }^{14}$. Briefly, curated

20 reference protein sequences of Types I, II, IIC and III R-M systems and Type IV REases

21 were downloaded from the data set 'gold standards' of REBASE ${ }^{65}$ (last accessed in

22 November 2016). All-against-all searches were performed for REase and MTase standard

23 protein sequences retrieved from REBASE using BLASTP v2.5.0+ (default settings, e value

$24<10^{-3}$ ). The resulting $e$ values were log-transformed and used for clustering into protein

25 families by Markov Clustering (MCL) v14-13766. Each protein family was aligned with MAFFT

$26 v 7.305 b^{67}$ using the E-INS-i option, 1,000 cycles of iterative refinement, and offset 0.

27 Alignments were visualized in SEAVIEW v4.6.1 $1^{68}$ and manually trimmed to remove poorly 
1 aligned regions at the extremities. Hidden Markov model (HMM) profiles were then built from

2 each multiple sequence alignment using the hmmbuild program from the HMMER v3.0

3 suite $^{69}$ (default parameters) (available at https://github.com/pedrocas81). Types I, II, and III

4 R-M systems were identified by searching genes encoding the MTase and REase

5 components at less than five genes apart.

6

7 CAAAAA motif abundance and exceptionality

8 We evaluated the exceptionality of the CAAAAA motif using R'MES ${ }^{36}$ v3.1.0

9 (http://migale.jouy.inra.fr/?q=rmes). This tool computes scores of exceptionality for k-mers of

10 length $I$, by comparing observed and expected counts under Markov models that take

11 sequence composition under consideration. R'MES outputs scores of exceptionality, which

12 are, by definition, obtained from $P$-values through the standard one-to-one probit

13 transformation. Analysis of motif abundance was performed with a previous developed

14 framework ${ }^{37}$ involving a multi-scale representation (MSR) of genomic signals. We created a

15 binary genomic signal for motif content, which was 1 at motif positions, and 0 otherwise. 50

16 length scales were used. Pruning parameter values were set to default and the $P$-value

17 threshold to $10^{-6}$.

19 Whole-genome multiple alignment and classification of CAAAAA positions

20 Whole-genome multiple alignment of 37 genomes (36 C. difficile isolates and C. difficile 630)

21 was produced by the progressiveMauve program ${ }^{70}$ v2.4.0 with default parameters. Since

22 progressiveMauve does not rely on annotations to guide the alignment, we first used the

23 Mauve Contig Mover $^{71}$ to reorder and reorient draft genome contigs according to the

24 reference genome of $C$. difficile 630. A core alignment was built after filtering and

25 concatenating locally collinear blocks (LCBs) of size $\geq 50$ bp using the stripSubsetLCBs

26 script (http://darlinglab.org/mauve/snapshots/2015/2015-01-09/linux-x64/). The lower value

27 chosen for LCB size accounts for the specific aim of maximizing the number of orthologous 
1 motifs detected. The XMFA output format of Mauve was converted to VCF format using

2 dedicated scripts, and VCFtools ${ }^{72}$ was used to parse positional variants (SNPs and indels).

3 Orthologous occurrences of the CAAAA $\underline{A}$ motif were defined if an exact match to the motif

4 was present in each of the 37 genomes (conserved orthologous positions), or if at least one

5 motif (and a maximum of $n-1$, with $n$ being the number of genomes) contained positional

6 polymorphisms (maximum of two SNPs or indels per motif) (variable orthologous positions).

7 Non-orthologous occurrences of CAAAA $\underline{A}$ were obtained from the whole genome alignment

8 before the extraction of LCBs. The former correspond to those situations where the

9 CAAAA $\underline{A}$ motif was absent in at least one genome. Typically, these correspond to regions

10 containing MGEs or unaligned repetitive regions.

\section{Identification of transcription factor binding sites, and transcription start sites}

13 Identification of transcription factor binding sites (TFBS) was performed by retrieving $C$.

14 difficile 630 regulatory sites in FASTA format from the RegPrecise database

15 (http://regprecise.lbl.gov ${ }^{73}$, last accessed July 2017). These were converted to PWMs using

16 in-house developed scripts. This led to a total of 21 PWMs pertaining to 14 distinct

17 transcription factor families (Supplementary Table 7b). Matches between these matrices and

18 C. difficile genomes was performed with $\mathrm{MAST}^{74}$ (default settings). MAST output was filtered

19 on the basis of $P$-value. Hits with $P$-value $<10^{-9}$ were considered positive, while hits

$20>10^{-5}$ were considered negative. Hits with intermediate $P$-values were only considered

21 positive if the $P$-value of the hit divided by the $P$-value of the worst positive hit was lower

22 than 100. For the CcpA, LexA, NrdR, and CodY (which have shorter binding sites), we

23 considered positive hits those with $P$-values $<10^{-8}$. Transcription start sites (TSSs) were

24 predicted with Parseq ${ }^{41}$ under the 'fast' speed option from multiple RNA-seq datasets (see

25 below). Transcription and breakpoint probabilities were computed using a background

26 expression level threshold of 0.1 and a score penalty of 0.05 . We kept only high-confidence

275 ' breakpoint hits, located at a maximum distance of $200 \mathrm{bp}$ from the nearest start codon. A 
$1 \pm 5$ bp window around the TSS was considered if only one single predicted value was

2 obtained; otherwise we considered an interval delimited by the minimum and maximum

3 values predicted by Parseq.

5 RNA sequencing, read alignment, and differential expression analysis

6 Purified RNA was extracted from three biological replicates of sporulating $(9,10.5 \mathrm{~h})$ and

7 exponential and stationary grown cultures of $C$. difficile $630 \Delta$ erm and $C$. difficile

$8630 \Delta e r m \Delta c a m A$, DNase-treated, ribosomal RNA-depleted, and converted to cDNA as

9 previously described ${ }^{44}$ (more details in Supplementary Methods). RNA sequencing was

10 performed on a HiSeq 2500 , yielding an average of $29.4( \pm 4.5, \mathrm{sd})$ million 100 -bp single-end

11 reads per sample (exponential and stationary growth timepoints) and $26.9( \pm 4.3, \mathrm{sd})$ million

12 150-bp paired-end reads per sample (sporulation time points). Read quality was checked

13 using FastQC v0.11.5 (http://www.bioinformatics.babraham.ac.uk/projects/fastac). We used

14 Trimmomatic $^{75}$ v0.39 to remove adapters and low-quality reads (parameters: PE, -phred33,

15 ILLUMINACLIP:<adapters.fa>:2:30:10:8:True, SLIDINGWINDOW:4:15, LEADING:20

16 TRAILING:20, MINLEN:50). Subsequently, rRNA sequences were filtered from the data set

17 using SortMeRNA ${ }^{76}$ v2.1, based on the SILVA 16s and 23s rRNA databases ${ }^{77}$, and Rfam 5s

18 rRNA database ${ }^{78}$. The resulting non-rRNA reads were mapped to the $C$. difficile 630

19 reference genome using BWA-MEM v0.7.17-r1198 ${ }^{79}$. The resulting bam files were sorted

20 and indexed with Samtools ${ }^{80}$ v1.9, and read assignment was performed with featureCounts ${ }^{81}$

21 v1.6.4 (excluding multi-mapping and multi-overlapping reads). A gene was included for

22 differential expression analysis if it had more than one count in all samples. Normalization

23 and differential expression testing were performed using the Bioconductor package DESeq2

24 v1.18.1 $1^{82}$. Genes with a false discovery rate $(F D R)<0.05$ and $\left|\log _{2} F C\right|>1$ were called as

25 differentially expressed. Functional classification of genes was performed using the DAVID

26 online database (https://david.ncifcrf.gov) ${ }^{83}$ with Fisher's exact test enrichment statistics, a

27 Benjamini-Hochberg corrected $P$-value cutoff of 0.05 , and FDR $<0.05$. The reproducibility of 
1 DAVID's functional classification was tested with Blast2GO v5.2 ${ }^{84}$ and Panther v14 ${ }^{85}$. Briefly,

2 for Blast2GO, we ran BLASTX searches of the $C$. difficile 630 genome against the entire

3 GenBank bacterial protein database (as of 09/2018). The output, in XML format, was loaded

4 into Blast2GO, and mapping, annotation and enrichment analysis was performed as

5 indicated (http://docs.blast2go.com/user-manual/quick-start/). For Panther, we downloaded

6 the most recent HMM library

7 (ftp.pantherdb.org/hmm scoring/13.1/PANTHER13.1 hmmscoring.tgz), and annotated our

8 C. difficile 630 protein set with pantherScore2.1.pl. Both input and background gene lists

9 were formatted to the Panther Generic Mapping File type, as described in the website

10 (http://www.pantherdb.org). To assess the significance of the intersection between multiple

11 datasets of differentially expressed genes (typically observed during $C$. difficile colonization

12 and infection), we collected gene-expression data from in vivo and in vitro studies ${ }^{59-61}$, in

13 which key factors for gut colonization (e.g., time post-infection, antibiotic exposure, and

14 spatial structure (planktonic, biofilm growth)) were tested. Differentially expressed genes

15 were called under the same conditions as described above. Statistical analyses and

16 graphical representation of multi-set intersections was performed with the $\mathrm{R}$ package

17 SuperExactTest ${ }^{86}$.

18 


\section{References}

2 1. Smits WK, Lyras D, Lacy DB, Wilcox MH, Kuijper EJ. Clostridium difficile infection. Nat. Rev. Dis. Primers 2, 16020 (2016).

2. Deakin LJ, et al. The Clostridium difficile spoOA gene is a persistence and transmission factor. Infect. Immun. 80, 2704-2711 (2012).

3. Paredes-Sabja D, Shen A, Sorg JA. Clostridium difficile spore biology: sporulation, germination, and spore structural proteins. Trends Microbiol. 22, 406-416 (2014).

4. Seekatz AM, Young VB. Clostridium difficile and the microbiota. J. Clin. Invest. 124, 41824189 (2014).

5. Zidaric V, Rupnik M. Sporulation properties and antimicrobial susceptibility in endemic and rare Clostridium difficile PCR ribotypes. Anaerobe 39, 183-188 (2016).

6. Collins $\mathrm{J}$, et al. Dietary trehalose enhances virulence of epidemic Clostridium difficile. Nature 553, 291-294 (2018).

7. Lanis JM, Barua S, Ballard JD. Variations in TcdB activity and the hypervirulence of emerging strains of Clostridium difficile. PLoS Pathog. 6, e1001061 (2010).

8. Valiente E, Cairns MD, Wren BW. The Clostridium difficile PCR ribotype 027 lineage: a pathogen on the move. Clin. Microbiol. Infect. 20, 396-404 (2014).

9. Lessa FC, et al. Burden of Clostridium difficile infection in the United States. N. Engl. J. Med. 372, 825-834 (2015).

10. Sebaihia $\mathrm{M}$, et al. The multidrug-resistant human pathogen Clostridium difficile has a highly mobile, mosaic genome. Nat. Genet. 38, 779-786 (2006).

11. He $\mathrm{M}$, et al. Emergence and global spread of epidemic healthcare-associated Clostridium difficile. Nat. Genet. 45, 109-113 (2013).

12. Herbert M, O'Keeffe TA, Purdy D, Elmore M, Minton NP. Gene transfer into Clostridium difficile CD630 and characterisation of its methylase genes. FEMS Microbiol. Lett. 229, 103110 (2003).

13. van Eijk E, et al. Complete genome sequence of the Clostridium difficile laboratory strain 630Deltaerm reveals differences from strain 630, including translocation of the mobile element CTn5. BMC Genomics 16, 31 (2015).

14. Oliveira PH, Touchon M, Rocha EP. The interplay of restriction-modification systems with mobile genetic elements and their prokaryotic hosts. Nucleic Acids Res. 42, 10618-10631 (2014).

15. Oliveira $\mathrm{PH}$, Touchon $\mathrm{M}$, Rocha EP. Regulation of genetic flux between bacteria by restriction-modification systems. Proc. Natl. Acad. Sci. USA 113, 5658-5663 (2016).

16. Casadesus J, Low D. Epigenetic gene regulation in the bacterial world. Microbiol. Mol. Biol. Rev. 70, 830-856 (2006).

17. Low DA, Weyand NJ, Mahan MJ. Roles of DNA adenine methylation in regulating bacterial gene expression and virulence. Infect. Immun. 69, 7197-7204 (2001). 
18. Cohen NR, et al. A role for the bacterial GATC methylome in antibiotic stress survival. Nat. Genet. 48, 581-586 (2016).

19. Manso AS, et al. A random six-phase switch regulates pneumococcal virulence via global epigenetic changes. Nat. Commun. 5, 5055 (2014).

20. Atack JM, et al. A biphasic epigenetic switch controls immunoevasion, virulence and niche adaptation in non-typeable Haemophilus influenzae. Nat. Commun. 6, 7828 (2015).

21. Wion D, Casadesus J. N6-methyl-adenine: an epigenetic signal for DNA-protein interactions. Nat. Rev. Microbiol. 4, 183-192 (2006).

22. Flusberg BA, et al. Direct detection of DNA methylation during single-molecule, real-time sequencing. Nat. Methods 7, 461-465 (2010).

23. Beaulaurier J, Schadt EE, Fang G. Deciphering bacterial epigenomes using modern sequencing technologies. Nat. Rev. Genet. 20, 157-172 (2019).

24. Fang G, et al. Genome-wide mapping of methylated adenine residues in pathogenic Escherichia coli using single-molecule real-time sequencing. Nat. Biotechnol. 30, 1232-1239 (2012).

25. Murray IA, et al. The methylomes of six bacteria. Nucleic Acids Res. 40, 11450-11462 (2012).

26. Davis BM, Chao MC, Waldor MK. Entering the era of bacterial epigenomics with single molecule real time DNA sequencing. Curr. Opin. Microbiol. 16, 192-198 (2013).

27. Smits WK. Hype or hypervirulence: a reflection on problematic C. difficile strains. Virulence 4, 592-596 (2013).

28. Roberts RJ, et al. A nomenclature for restriction enzymes, DNA methyltransferases, homing endonucleases and their genes. Nucleic Acids Res. 31, 1805-1812 (2003).

29. Wust J, Sullivan NM, Hardegger U, Wilkins TD. Investigation of an outbreak of antibioticassociated colitis by various typing methods. J. Clin. Microbiol. 16, 1096-1101 (1982).

30. Dembek $\mathrm{M}$, et al. High-throughput analysis of gene essentiality and sporulation in Clostridium difficile. MBio 6, e02383 (2015).

31. Barra-Carrasco J, Paredes-Sabja D. Clostridium difficile spores: a major threat to the hospital environment. Future Microbiol. 9, 475-486 (2014).

32. $\mathrm{Ng} \mathrm{YK}$, et al. Expanding the repertoire of gene tools for precise manipulation of the Clostridium difficile genome: allelic exchange using pyrE alleles. PLOS ONE 8, e56051 (2013).

33. Donnelly ML, Fimlaid KA, Shen A. Characterization of Clostridium difficile spores lacking either SpoVAC or dipicolinic acid synthetase. J. Bacteriol. 198, 1694-1707 (2016).

34. Pogliano J, et al. A vital stain for studying membrane dynamics in bacteria: a novel mechanism controlling septation during Bacillus subtilis sporulation. Mol. Microbiol. 31, 11491159 (1999).

35. Shen A, Fimlaid KA, Pishdadian K. Inducing and quantifying Clostridium difficile spore formation. Methods Mol. Biol. 1476, 129-142 (2016).

36. Schbath S, Hoebeke M. R'MES: a tool to find motifs with a significantly unexpected frequency in biological sequences. In: Advances in genomic sequence analysis and pattern discovery $\left(e^{\wedge}(e d s\right.$ L. Elnitsk i OP, and L. Welch). World Scientific (2011). 
37. Knijnenburg TA, et al. Multiscale representation of genomic signals. Nat. Methods 11, 689694 (2014).

38. Lim HN, van Oudenaarden A. A multistep epigenetic switch enables the stable inheritance of DNA methylation states. Nat. Genet. 39, 269-275 (2007).

39. Ardissone $\mathrm{S}$, et al. Cell cycle constraints and environmental control of local DNA hypomethylation in alpha-Proteobacteria. PLoS Genet. 12, e1006499 (2016).

40. Cota I, Bunk B, Sproer C, Overmann J, Konig C, Casadesus J. OxyR-dependent formation of DNA methylation patterns in OpvABOFF and OpvABON cell lineages of Salmonella enterica. Nucleic Acids Res. 44, 3595-3609 (2016).

41. Mirauta $B$, Nicolas $P$, Richard $H$. Parseq: reconstruction of microbial transcription landscape from RNA-Seq read counts using state-space models. Bioinformatics 30, 1409-1416 (2014).

42. Moxon R, Bayliss C, Hood D. Bacterial contingency loci: the role of simple sequence DNA repeats in bacterial adaptation. Annu. Rev. Genet. 40, 307-333 (2006).

43. Sater MR, et al. DNA methylation assessed by SMRT Sequencing Is linked to mutations in Neisseria meningitidis isolates. PLOS ONE 10, e0144612 (2015).

44. Fimlaid KA, et al. Global analysis of the sporulation pathway of Clostridium difficile. PLoS Genet. 9, e1003660 (2013).

45. Pishdadian K, Fimlaid KA, Shen A. SpollID-mediated regulation of sigmaK function during Clostridium difficile sporulation. Mol. Microbiol. 95, 189-208 (2015).

46. Pereira FC, et al. The spore differentiation pathway in the enteric pathogen Clostridium difficile. PLoS Genet. 9, e1003782 (2013).

47. Saujet L, Pereira FC, Henriques AO, Martin-Verstraete I. The regulatory network controlling spore formation in Clostridium difficile. FEMS Microbiol Lett 358, 1-10 (2014).

48. Pishdadian K, Fimlaid KA, Shen A. SpollID-mediated regulation of sigmaK function during Clostridium difficile sporulation. Mol Microbiol 95, 189-208 (2015).

49. Serrano $\mathrm{M}$, et al. A recombination directionality factor controls the cell type-specific activation of sigmaK and the fidelity of spore development in Clostridium difficile. PLoS Genet. 12, e1006312 (2016).

50. Saujet L, et al. Genome-wide analysis of cell type-specific gene transcription during spore formation in Clostridium difficile. PLoS Genet. 9, e1003756 (2013).

51. Rosenbusch KE, Bakker D, Kuijper EJ, Smits WK. C. difficile 630Deltaerm Spo0A regulates sporulation, but does not contribute to toxin production, by direct high-affinity binding to target DNA. PLOS ONE 7, e48608 (2012).

52. Saujet L, et al. Genome-wide analysis of cell type-specific gene transcription during spore formation in Clostridium difficile. PLoS Genet 9, e1003756 (2013).

53. Fimlaid KA, et al. Global analysis of the sporulation pathway of Clostridium difficile. PLoS Genet 9, e1003660 (2013).

54. Fimlaid KA, Jensen O, Donnelly ML, Siegrist MS, Shen A. Regulation of Clostridium difficile Spore Formation by the SpolIQ and SpollIA Proteins. PLoS Genet 11, e1005562 (2015).

55. Ribis JW, Fimlaid KA, Shen A. Differential requirements for conserved peptidoglycan remodeling enzymes during Clostridioides difficile spore formation. Mol Microbiol 110, 370 389 (2018). 
1

56. Chen X, et al. A mouse model of Clostridium difficile-associated disease. Gastroenterology 135, 1984-1992 (2008).

57. Theriot CM, Koumpouras CC, Carlson PE, Bergin, II, Aronoff DM, Young VB. Cefoperazonetreated mice as an experimental platform to assess differential virulence of Clostridium difficile strains. Gut Microbes 2, 326-334 (2011).

58. McKee RW, Aleksanyan N, Garrett EM, Tamayo R. Type IV pili promote Clostridium difficile adherence and persistence in a mouse model of infection. Infect. Immun. 86, (2018).

59. Maldarelli GA, et al. Type IV pili promote early biofilm formation by Clostridium difficile. Pathog. Dis. 74, (2016).

60. Jenior ML, Leslie JL, Young VB, Schloss PD. Clostridium difficile colonizes alternative nutrient niches during infection across distinct murine gut microbiomes. mSystems 2, (2017).

61. Fletcher JR, Erwin S, Lanzas C, Theriot CM. Shifts in the gut metabolome and Clostridium difficile transcriptome throughout colonization and infection in a mouse model. mSphere $\mathbf{3}$, (2018).

62. Lewis BB, Pamer EG. Microbiota-Based Therapies for Clostridium difficile and AntibioticResistant Enteric Infections. Annual review of microbiology 71, 157-178 (2017).

63. Abt MC, McKenney PT, Pamer EG. Clostridium difficile colitis: pathogenesis and host defence. Nat Rev Microbiol 14, 609-620 (2016).

64. Sanchez-Romero MA, Cota I, Casadesus J. DNA methylation in bacteria: from the methyl group to the methylome. Curr. Opin. Microbiol. 25, 9-16 (2015).

65. Roberts RJ, Vincze T, Posfai J, Macelis D. REBASE--a database for DNA restriction and modification: enzymes, genes and genomes. Nucleic Acids Res. 43, D298-299 (2015).

66. Enright AJ, Van Dongen S, Ouzounis CA. An efficient algorithm for large-scale detection of protein families. Nucleic Acids Res. 30, 1575-1584 (2002).

67. Katoh K, Standley DM. MAFFT: iterative refinement and additional methods. Methods Mol. Biol. 1079, 131-146 (2014).

68. Gouy M, Guindon S, Gascuel O. SeaView version 4: A multiplatform graphical user interface for sequence alignment and phylogenetic tree building. Mol. Biol. Evol. 27, 221-224 (2010).

69. Finn RD, Clements J, Eddy SR. HMMER web server: interactive sequence similarity searching. Nucleic Acids Res. 39, W29-37 (2011).

70. Darling AE, Mau B, Perna NT. progressiveMauve: multiple genome alignment with gene gain, loss and rearrangement. PLOS ONE 5, e11147 (2010).

71. Rissman Al, Mau B, Biehl BS, Darling AE, Glasner JD, Perna NT. Reordering contigs of draft genomes using the Mauve aligner. Bioinformatics 25, 2071-2073 (2009).

72. Danecek P, et al. The variant call format and VCFtools. Bioinformatics 27, 2156-2158 (2011).

73. Novichkov PS, et al. RegPrecise 3.0--a resource for genome-scale exploration of transcriptional regulation in bacteria. BMC Genomics 14, 745 (2013).

74. Bailey TL, Gribskov M. Combining evidence using p-values: application to sequence homology searches. Bioinformatics 14, 48-54 (1998). 
1

2

3

4

5

6

75. Bolger AM, Lohse M, Usadel B. Trimmomatic: a flexible trimmer for Illumina sequence data. Bioinformatics 30, 2114-2120 (2014).

76. Kopylova E, Noe L, Touzet H. SortMeRNA: fast and accurate filtering of ribosomal RNAs in metatranscriptomic data. Bioinformatics 28, 3211-3217 (2012).

77. Quast C, et al. The SILVA ribosomal RNA gene database project: improved data processing and web-based tools. Nucleic Acids Res. 41, D590-596 (2013).

78. Griffiths-Jones S, Bateman A, Marshall M, Khanna A, Eddy SR. Rfam: an RNA family database. Nucleic Acids Res. 31, 439-441 (2003).

79. Li H, Durbin R. Fast and accurate short read alignment with Burrows-Wheeler transform. Bioinformatics 25, 1754-1760 (2009).

80. Li H, et al. The Sequence Alignment/Map format and SAMtools. Bioinformatics 25, 2078-2079 (2009).

81. Liao Y, Smyth GK, Shi W. featureCounts: an efficient general purpose program for assigning sequence reads to genomic features. Bioinformatics 30, 923-930 (2014).

82. Love MI, Huber W, Anders S. Moderated estimation of fold change and dispersion for RNAseq data with DESeq2. Genome Biol. 15, 550 (2014).

83. Huang DW, et al. DAVID Bioinformatics Resources: expanded annotation database and novel algorithms to better extract biology from large gene lists. Nucleic Acids Res. 35, W169-175 (2007).

84. Conesa A, Gotz S, Garcia-Gomez JM, Terol J, Talon M, Robles M. Blast2GO: a universal tool for annotation, visualization and analysis in functional genomics research. Bioinformatics 21, 3674-3676 (2005).

85. Mi H, Muruganujan A, Ebert D, Huang X, Thomas PD. PANTHER version 14: more genomes, a new PANTHER GO-slim and improvements in enrichment analysis tools. Nucleic Acids Res. 47, D419-D426 (2019).

86. Wang M, Zhao Y, Zhang B. Efficient test and visualization of multi-set intersections. Sci. Rep. 5, 16923 (2015).

87. Bordeleau E, Fortier LC, Malouin F, Burrus V. c-di-GMP turn-over in Clostridium difficile is controlled by a plethora of diguanylate cyclases and phosphodiesterases. PLOS Genet. 7 , e1002039 (2011).

88. Burton BM, Marquis KA, Sullivan NL, Rapoport TA, Rudner DZ. The ATPase SpollIE transports DNA across fused septal membranes during sporulation in Bacillus subtilis. Cell 131, 1301-1312 (2007). 


\section{$1 \quad$ Acknowledgements}

2 We acknowledge Dr. Richard J. Roberts (New England Biolabs, Inc. USA) for his expertise

3 and generous help with the prediction of R-M systems and orphan MTases in C. difficile

4 genomes using REBASE Tools, his critical reading, and for providing helpful

5 comments/suggestions. He was originally an author of this manuscript, however, as a

6 staunch supporter of the open access movement, he will not author a paper that is not open

7 access. We also acknowledge Dr. Eduardo P.C. Rocha (Institut Pasteur, Paris, France) for

8 critical reading and for providing helpful comments/suggestions. The work was primarily

9 funded by R01 GM114472 (G.F.) from the National Institutes of Health and Icahn Institute for

10 Genomics and Multiscale Biology. In addition, the work was funded by NIH grants R01

11 Al119145 (H.v.B and A.B.), R01 Al22232 (A.S.) and R01 Al107029 (R.T.) a Hirschl

12 Research Scholar award from the Irma T. Hirschl/Monique Weill-Caulier Trust (G.F.), a Pew

13 Scholar in the Biomedical Sciences grant from the Pew Charitable (A.S.). G.F. is a Nash

14 Family Research Scholar. A.S. holds an Investigators in the Pathogenesis of Infectious

15 Disease Award from the Burroughs Wellcome Fund. This work was also supported in part

16 through the computational resources and staff expertise provided by the Department of

17 Scientific Computing at the Icahn School of Medicine at Mount Sinai.

\section{Author Contributions}

20 G.F. conceived the hypothesis. A.S. and G.F. supervised the project. P.H.O. and G.F. 21 designed the computational methods. P.H.O., R.T., A.S., and G.F. designed the 22 experiments. P.H.O. performed most of the computational analyses and developed most of 23 the scripts supporting the analyses. J.W.R. performed the growth curves, microscopy 24 analyses (fluorescence and phase-contrast), analyses of cell length and sporulation stage, 25 isolated some of the RNA and processed it for qRT-PCR studies; qRT-PCR analyses of 26 sporulation genes. A.S. constructed the deletion and catalytic $\Delta$ camA mutants, performed 
1 complementation, isolated and processed the RNA for several of the RNA analyses and

2 performed many of the sporulation phenotypic assays. E.M.G. and D.T. performed the

3 animal infection experiment and analyzed the data under the supervision of R.T. A.Kim and

4 G.F. performed methylation motif discovery and refinement. O.S. and E.A.M. performed

5 qRT-PCR controls for RNA-seq analyses. O.S., E.A.M., G.D., M.L., C.B., N.Z., D.A., I.O.,

6 G.P., C.H., S.H., R.S., H.v.B. and A.S. contributed to the other experiments. G.D., I.O., and

7 R.S. designed and conducted SMRT sequencing. P.H.O., J.W.R., E.M.G., D.T., A.Kim.,

8 O.S., T.P., S.Z., E.A.M., M.T., S.B., A.A., A.B., R.J.B., R.T., E.E.S., R.S., H.B., A.Kasarskis.,

9 R.T., A.S. and G.F. analyzed the data. P.H.O., R.T., A.S., and G.F. wrote the manuscript

10 with additional information inputs from other co-authors. 


\section{Figure Legends}

2 Fig. 1. Methylomes of the 36 C. difficile strains. (a) Phylogenetic tree of the 36 C. difficile

3 strains colored by clade (hypervirulent, human and animal (HA) associated) and MLST

4 sequence type (ST). Heatmap depicting the landscape of methylated motifs per genome,

5 and their average interpulse duration (IPD) ratio. Asterisks refer to new motifs not previously

6 listed in the reference database REBASE. Methylated bases are underlined. The CAAAAA

7 motif was consistently methylated across isolates. Barplot indicates the number and types of

8 active MTases detected per genome. In Type IIC systems, MTase and REase are encoded

9 in the same polypeptide. (b) Representation of the $C$. difficile methylome. Shown are the

10 positions of all methylation motif sites in the reference genome of $C$. difficile 630 , colored

11 according to MTase type. Also shown are the average motif occurrences per genome

12 (across the 36 isolates). (c) \% of MTases detected according to type. (d) \% MTases

13 pertaining to complete R-M systems or without cognate REase (solitary). (e) Breakdown of

14 MTases by location: Integrative Mobile Elements (IMEs), Integrative Conjugative Elements

15 (ICEs), prophages, and other (within the chromosome). No hits were obtained in plasmids.

16 (f) Immediate genomic context of camA. The example shown (including coordinates) refers

17 to the reference genome of $C$. difficile $630 .+/$ - signs correspond to the sense and

18 antisense strands respectively. Vertical bars correspond to the distribution of the CAAAA $\underline{A}$

19 motif. CD2754: phosphodiesterase with a GGDEF domain (PF00990) and a cache domain

20 (PF02743); pts/ and ptsH belong to a phosphotransferase (PTS) system; CD2757: patatin-

21 like phospholipase (PF01734); CD2758 (camA): Type II MTase; CD2759: Rrf2-type

22 transcriptional regulator; CD2760: phosphodiesterase with a GGDEF domain (PF00990) and

23 a conserved EAL domain (PF00563) ${ }^{87}$; CD2761: N-acetylmuramoyl-L-alanine amidase;

24 CD2762: undecaprenyl diphosphate synthase.

26 Fig. 2. CamA modulates sporulation levels in $C$. difficile. (a) Spore purification efficiencies obtained from sporulating cells harvested 3 days after plating. The spore yield (arbitrary

28 units, a.u.), was determined by measuring the optical density at $600 \mathrm{~nm}$ of the resulting 
1 spore preparations and correcting for the volume of water in which spores were re-

2 suspended. (b) Phase-contrast microscopy after $20 \mathrm{~h}$ of sporulation induction. The $\triangle s p o 0 A$

3 strain was used as a negative control because it does not initiate sporulation ${ }^{2}$. Immature

4 phase-dark forespores are marked in pink, and mature phase-bright forespores and free

5 spores are shown in yellow and blue, respectively. Scale bar represents $5 \mu \mathrm{m}$. (c)

6 Morphological analysis of wild-type and $\Delta$ camA cells using fluorescent stains comparing 9

7 and $11 \mathrm{~h}$ following sporulation induction. The polar septum formed during asymmetric

8 division is visible using the FM4-64 membrane stain, while the chromosome that is pumped

9 into the forespore after polar septum formation can be seen as a bright foci using the

10 Hoechst 33342 nucleoid stain $^{34,88}$. FM4-64 staining also allows the engulfing membranes to

11 be visualized. As the mother cell-derived membrane fully encircles the forespore-derived

12 membrane, the FM4-64 signal becomes more intense around the forespore. When these

13 membranes undergo fission, the forespore becomes fully suspended in the mother cell

14 cytosol, and the FM4-64 and Hoechst stains are excluded. Yellow arrows show cells that are

15 undergoing asymmetric division (indicated by a flat polar septum); orange arrows show cells

16 that are in the process of engulfment (indicated by a curved polar septum); and blue arrows

17 show cells that have completed engulfment (indicated by bright membrane staining fully

18 surrounding the forespore). Barplots indicate the number of sporulating cells at different

19 stages of spore assembly in both wild-type and $\Delta$ camA cells. A minimum of 1000 cells per

20 replicate, per strain, and a minimum of two fields were scored for each strain at each

21 timepoint. * $P \leq 0.05,{ }^{* *} P<10^{-2}$, unpaired Student's t-test.

23 Fig. 3. Abundance, distribution, and conservation of CAAAA $\underline{A}$ motif sites. (a) Distribution of

24 CAAAA $\underline{A}$ motif sites in both strands of the reference C. difficile 630 genome and 25 corresponding genomic signal obtained by MSR. Letters (A-E) represent regions with 26 particularly high abundance of CAAAA $\underline{A}$ motifs at scales above 20 , i.e., typically above the 27 single gene level (median gene size in $C$. difficile $=0.78 \mathrm{~kb}$ ) (see Supplementary Table $6 \mathrm{~d}$ 28 for genes contained in each of the regions). Relation between MSR scale and segment 
1 length is also shown. The significant fold-change (SFC) corresponds to the fold-change $\left(\log _{2}\right.$

2 ratio) between observed and randomly expected overlap statistically significant at $P=10^{-6}$.

3 Heatmap layers correspond to the number of orthologous conserved (no SNPs/indels,

4 green-shaded) and orthologous variable (with SNPs/indels) $\mathrm{CA}_{5}$ motif positions. (b) Whole

5 genome alignment of 37 C. difficile genomes (36 isolates $+C$. difficile 630 as reference) was

6 performed using Mauve. We defined an orthologous occurrence of the CAAAA $\underline{A}$ motif (black

7 triangles) if an exact match to the motif was present in each of the 37 genomes (conserved,

8 blue-shaded regions), or if at least one motif (and a maximum of $n-1$, being $n$ the number of

9 genomes) contained positional polymorphisms (maximum of two SNPs or indels per motif)

10 (variable, green-shaded regions). Non-orthologous occurrences of CAAAA $\underline{A}$ are indicated as

11 orange-shaded regions. The results are shown in Fig. 3a in the form of heatmaps.

12 Numbering in scheme is based on mapping location. (c) DAVID enrichment analysis of the

13 genes containing intragenic and regulatory (100 bp upstream the start codon) orthologous

14 variable CAAAA $\underline{A}$ motif sites. We considered a Fisher's exact test enrichment statistics, a

15 Benjamini-Hochberg corrected $P$-value cutoff of 0.05 , and a false discovery rate $(\mathrm{FDR})<$

$16 \quad 0.05$.

18 Fig. 4. Distribution of non-methylated CAAAAA motif sites, and overlap with transcription 19 factor binding sites (TFBS) and transcription start sites (TSS). (a) Number of C. difficile 20 isolates for which non-methylated CAAAAA motif sites were detected at a given 21 chromosome position (coordinates are relative to the reference genome of $C$. difficile 630).

22 Peak colors correspond to orthologous (conserved and variant) and non-orthologous 23 CAAAA $\underline{A}$ positions. Some of the major peaks of non-methylated CAAAA $\underline{A}$ positions were 24 found to overlap with TFBS (e.g., CodY, XylR) and TSS. (b) Genetic regions for which overlap was observed between highly conserved non-methylated CAAAA $\underline{A}$ motif sites (red ovals) and TFs (CodY and XyIR, shown in blue). Other examples of conserved non-

27 methylated CAAAA $\underline{A}$ motif sites are illustrated in Supplementary Fig. 7b. (c) \% CAAAA $\underline{A}$ 28 motif sites (non-methylated and methylated) overlapping CodY and XyIR for each C. difficile 
1 isolate. (d) Example of a chromosomal region in which non-methylated CAAAA $\underline{A}$ motifs

2 overlap a TSS (shown as arrow). (e) \% CAAAA $\underline{A}$ motifs (non-methylated (NM) and

3 methylated $(\mathrm{M})$ ) overlapping TSSs for each $C$. difficile isolate. (f) \% mutated reads (SNPs +

4 indels) in CAAAA $\underline{A}$ and $\mathrm{K}(\mathrm{G}$ or T)AAAAA motifs for $M$. tuberculosis (MT), E. coli (EC), $C$.

5 difficile (CD) and $H$. pylori (HP). AAAAAA was not considered as control motif as it would

6 theoretically be more error-prone. ${ }^{* * *} P<10^{-3}$, Mann-Whitney-Wilcoxon test.

8 Fig. 5. Gene expression analysis. (a) Heatmap of 161 genes in three replicates of $C$. difficile

9630 compared to equal number of replicates of $C$. difficile $630 \Delta$ camA and that are enriched

10 for the GO terms shown in boxes and detailed in Supplementary Table 8c. The $Z$ score

11 reflects the degree of down- $(Z$ score $<0)$ or up- $(Z$ score $>0)$ regulation, computed by

12 subtracting the mean of the log-transformed expression values and dividing by the standard

13 deviation for each gene over all samples scored. (b) Schematic illustrating the sequence of

14 sporulation sigma factor gene transcription and protein activation coupled to morphological

15 changes during sporulation. (c) Comparison of relative transcript levels in wild type and

$16 \Delta$ camA as determined by qRT-PCR for sporulation sigma factor genes and representative

17 genes in the regulons of sporulation-specific sigma factors at 9 and $11 \mathrm{~h}$ after sporulation

18 induction. It should be noted that the primers for sigK amplify a region prior to the sigK

19 excision site $^{49}$. Statistical significance was determined by one-way ANOVA and Tukey's test

20 for multiple comparisons $\left({ }^{*} P \leq 0.05\right.$, ${ }^{* *} P<10^{-2}$, ${ }^{* * *} P<10^{-3}$, $\left.{ }^{* * *} P<10^{-4}\right)$.

22 Fig. 6. In vivo and additional functional impacts of the $\triangle$ camA mutation. (a) Kinetics of 23 infection in mice $(n=12)$ following inoculation with a sub-lethal amount $\left(10^{5}\right.$ spores) of wild

24 type (WT) C. difficile 630 $\Delta$ erm, MTase mutant $\triangle c a m A$, and complement $\triangle c a m A-C$. Dotted

25 line indicates the limit of detection. $\log _{10}$-transformed data from each time point were

26 analyzed by ANOVA. ${ }^{*} P<0.05$, ${ }^{\star \star \star \star} P<10^{-4}$. (b) Kaplan-Meier survival curves for

27 clindamycin-treated golden Syrian hamsters $(n=6)$ infected with $10^{3}$ spores of either wild 
1 type (WT) C. difficile 630 $\Delta$ erm, MTase mutant $\triangle$ camA, and complement $\Delta c a m A-C$. (c)

2 Representative phase-contrast images of vegetative wild type, $\triangle \operatorname{cam} A, \Delta \operatorname{cam} A-C$, and

$3 \Delta$ camA/N165A, grown in BHIS broth (scale bar $5 \mu \mathrm{m}$ ). (d) Comparison of cell length. A

4 minimum of 400 cells were measured for each strain at mid-log and stationary phase, and a

5 minimum of 1,600 cells were analyzed during sporulation at the indicated timepoints. All cell

6 length measurements were conducted on three independent biological replicates. Statistical

7 significance was determined by one-way ANOVA and Tukey's test for multiple comparisons.

8 (e) Significance of overlap between multiple datasets of DE genes. Comparisons were

9 performed between DE genes called in this study for each time point (blue-shaded) and

10 those from Maldarelli et al. ${ }^{59}$ (green-shaded). The latter corresponds to $C$. difficile DE genes

11 in conditions favoring biofilm formation compared to growth on a plate or planktonic form.

12 Color intensities of the outermost layer represent the $P$-value significance of the

13 intersections (3,896 genes were used as background). The height of the corresponding bars

14 is proportional to the number of common genes in the intersection (indicated at the top of the

15 bars for pairwise comparisons between the different studies). (f) Biofilm production as

16 measured by crystal violet staining absorbance at $570 \mathrm{~nm}$. Error bars correspond to

17 standard error of the mean of three independent experiments, with each strain assayed in

18 quadruplicate in each experiment. ${ }^{*} P<0.05$, ${ }^{* *} P<10^{-2}$, ${ }^{\star \star *} P<10^{-3}$, unpaired Student's t-

19 test. 
a

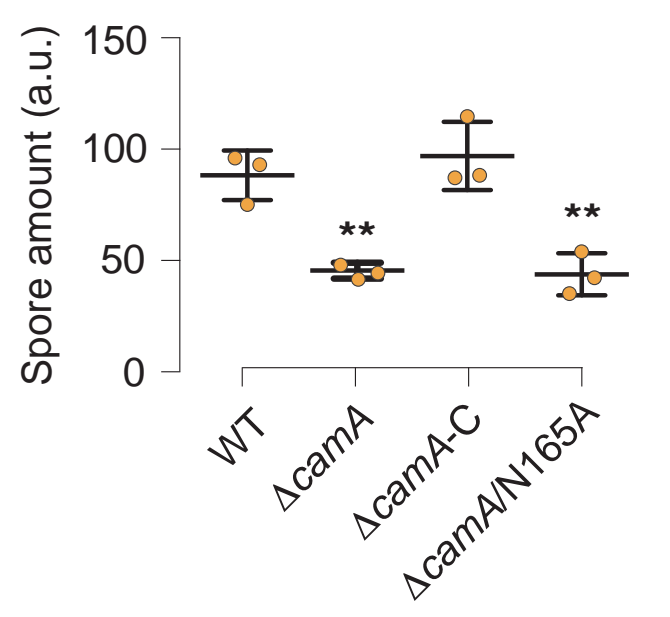

C
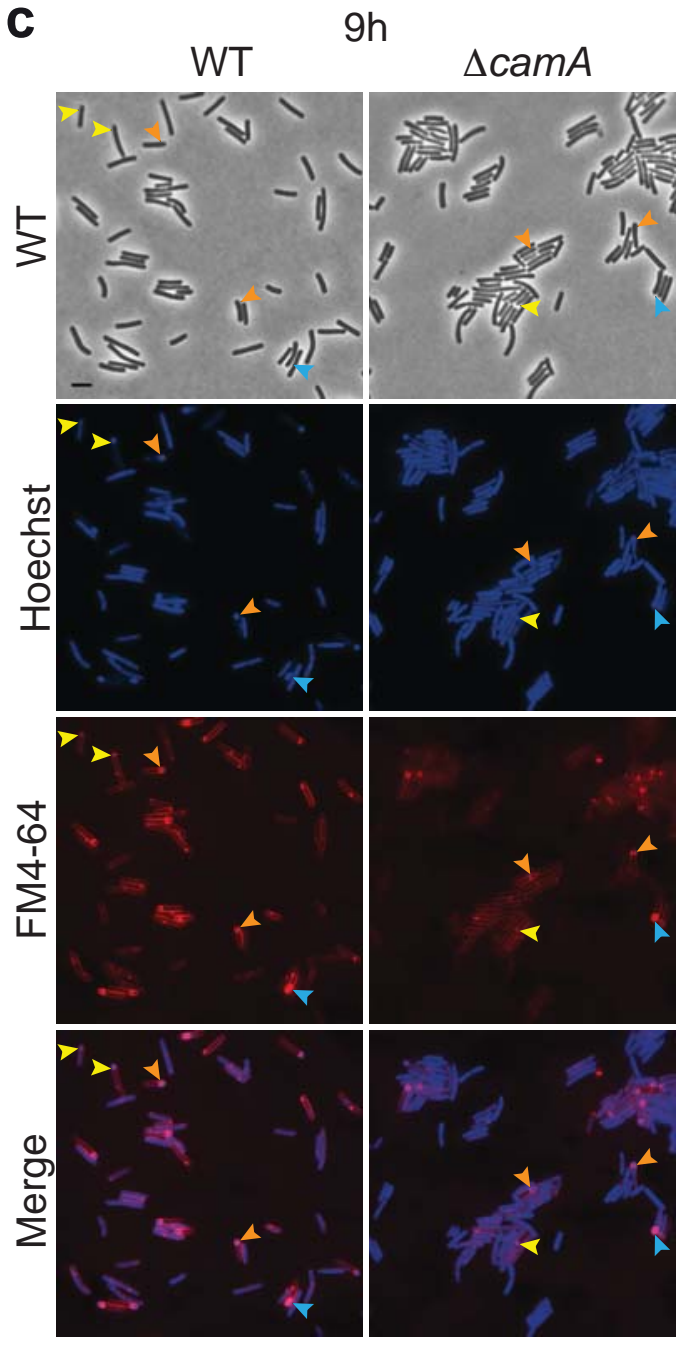

$<$ Asymmetric division $<$ Engulfment initiated $<$ Engulfment completed

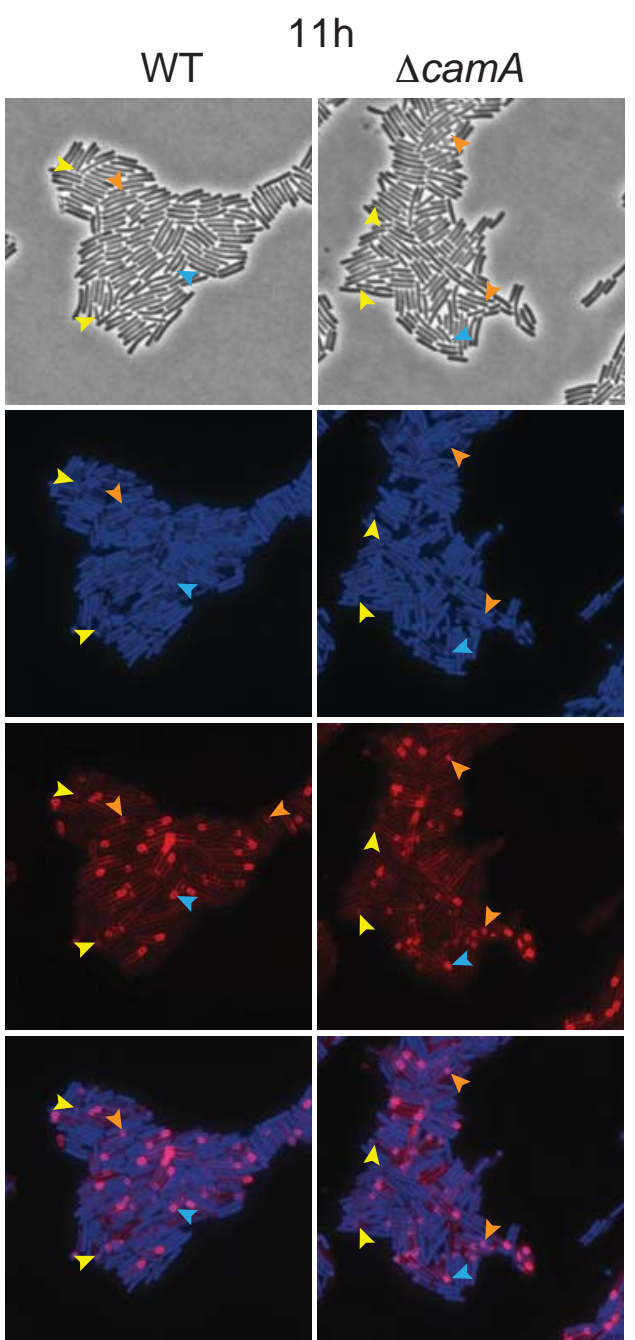

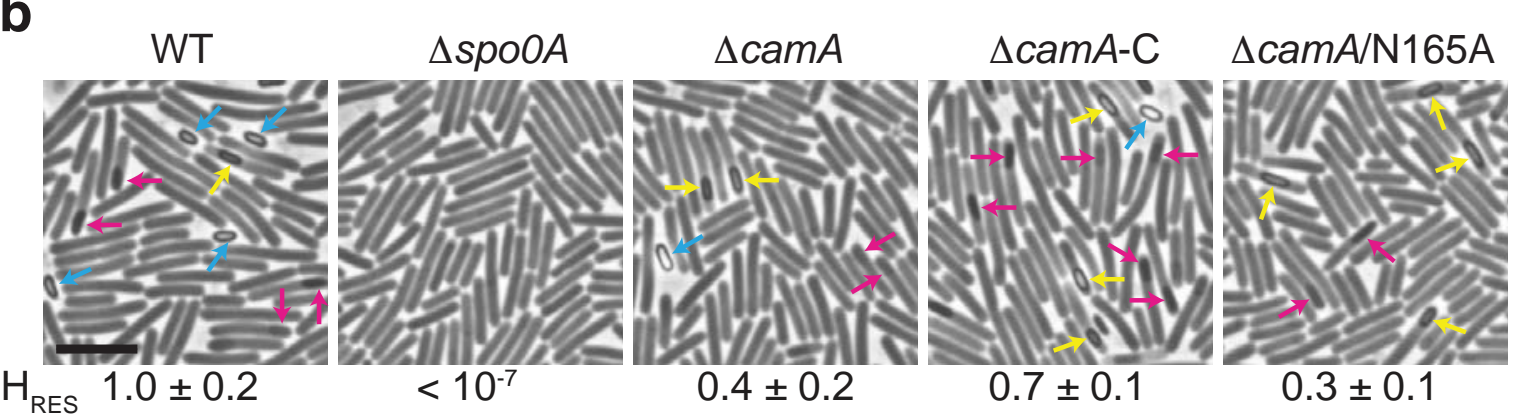

$\rightarrow$ Phase-dark forespore $\rightarrow$ Phase-bright forespore $\rightarrow$ Phase-bright spore
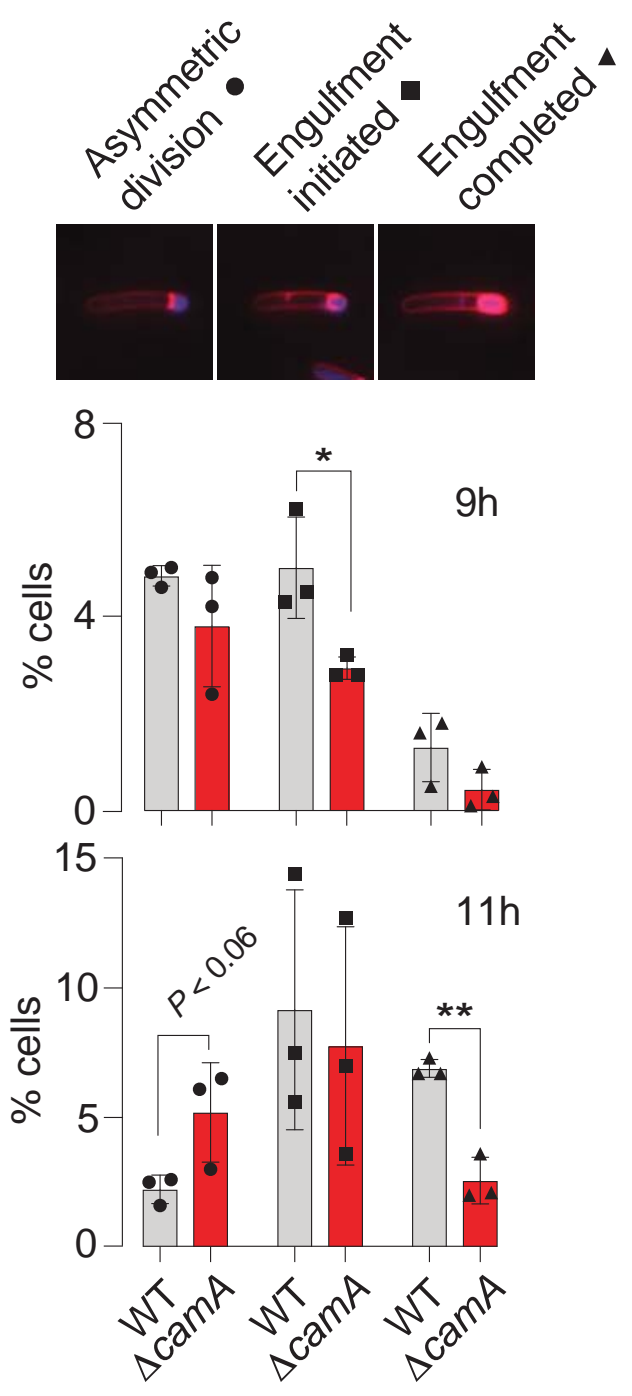


\section{$\mathbf{a}$}

Non-methylated

CAAAA $\underline{A}$

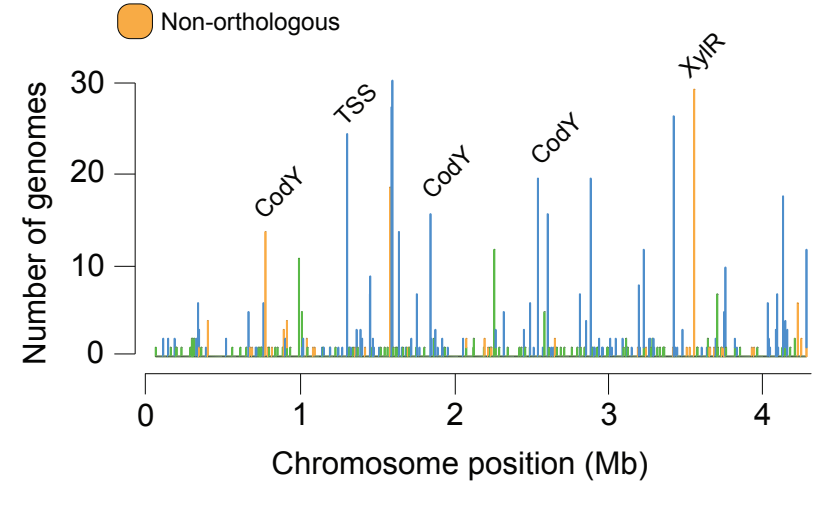

b CodY $-786,216 \quad$ CodY $-2,551,064$
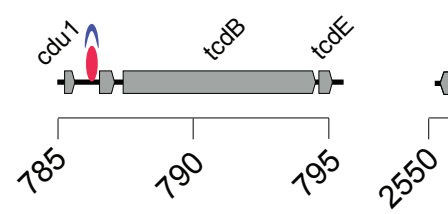

CodY - 1,853,965

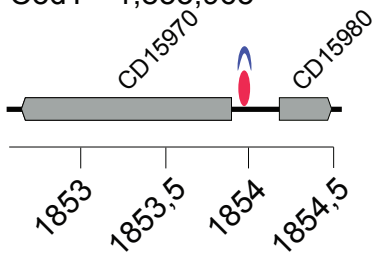

Chromosome position (kb)
C
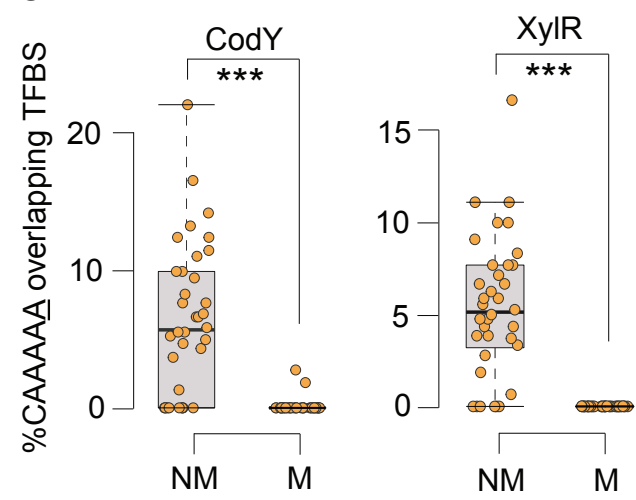

f

f CAAAAA positions

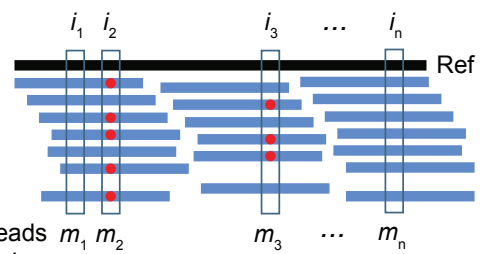

Mutated reads $m_{1} m_{2}$ at position $i$

Total reads at position $i$

SNP or indel •

$$
\begin{array}{ccccc}
t_{1} & t_{2} & t_{3} & \cdots & t_{\mathrm{n}}
\end{array}
$$

$\%$ mutated reads $=\frac{\sum_{\mathrm{i}} m_{\mathrm{i}}}{\sum_{\mathrm{i}} t_{\mathrm{i}}}$
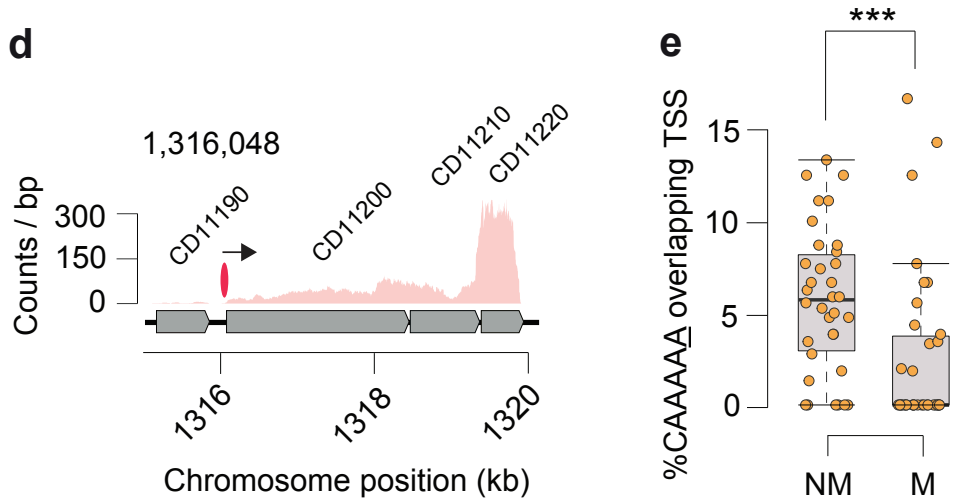
